\title{
Paradoxical effects of JZL184, an inhibitor of monoacylglycerol lipase, on bone remodelling in healthy and cancer-bearing mice
}

\author{
Silvia Marino ${ }^{\mathrm{a}, \mathrm{b}}$, Daniëlle de Ridder ${ }^{\mathrm{a}}$, Ryan T. Bishop ${ }^{\mathrm{a}}$, Nathalie Renema ${ }^{\mathrm{c}}$, Marco Ponzetti ${ }^{\mathrm{d}}$, \\ Antonia Sophocleous ${ }^{\text {e,f }}$, Mattia Capulli d , Abdullah Aljeffery ${ }^{\mathrm{a}}$, Giovana Carrasco ${ }^{\mathrm{a}}$, Marianela Dalghi Gens ${ }^{\mathrm{g}}$, \\ Asim Khogeer a,b, Stuart H. Ralston e , Jürg Gertsch ${ }^{\mathrm{g}}$, Francois Lamoureux ${ }^{\mathrm{c}}$, Dominique Heymann ${ }^{\mathrm{a}, \mathrm{h}, 1}$, \\ Nadia Rucci ${ }^{\mathrm{a}, \mathrm{d}, 1}$, Aymen I. Idris ${ }^{\mathrm{a}, \mathrm{b}, *}$ \\ a Department of Oncology and Metabolism, University of Sheffield, Medical School, Beech Hill Road, Sheffield S10 2RX, UK \\ ${ }^{\mathrm{b}}$ Bone and Cancer Group, Edinburgh Cancer Research Centre, MRC Institute of Genetics and Molecular Medicine, University of Edinburgh, EH4 2XR, UK \\ c INSERM, U1238, University of Nantes, Faculty of Medicine, 1 rue Gaston Veil, 44035 Nantes, Cedex 1, France \\ ${ }^{\mathrm{d}}$ University of L'Aquila, Department of Biotechnological and Applied Clinical Sciences, L'Aquila, Italy \\ e Rheumatic disease unit, MRC Institute of Genetics and Molecular Medicine, University of Edinburgh, Crewe Road, Edinburgh EH4 2XU, UK \\ ${ }^{\mathrm{f}}$ Department of Life Sciences, School of Sciences, European University Cyprus, 6 Diogenes Street, 1516 Nicosia, Cyprus \\ ${ }^{g}$ Institute of Biochemistry and Molecular Medicine, University of Bern, Switzerland \\ h INSERM, U1232, CRCINA, Institut de Cancérologie de l'Ouest, University of Nantes, Université d'Angers, Blvd Jacques Monod, 44805 Saint-Herblain, France
}

\section{A R T I C L E I N F O}

\section{Article history:}

Received 13 December 2018

Received in revised form 21 May 2019

Accepted 21 May 2019

Available online 29 May 2019

\section{Keywords:}

MAGL

Cannabinoid

Breast

Prostate

Bone

Cancer

Sarcoma

Osteolysis

Osteoclast

Metastasis

\begin{abstract}
A B S T R A C T
Background: Cancer-associated bone disease is a serious complication in bone sarcomas and metastatic carcinomas of breast and prostate origin. Monoacylglycerol lipase (MAGL) is an enzyme of the endocannabinoid system, and is responsible for the degradation of the most abundant endocannabinoid in bone, 2-arachidonoyl glycerol (2AG).

Methods: The effects of the verified MAGL inhibitor on bone remodelling were assessed in healthy mice and in mouse models of bone disease caused by prostate and breast cancers and osteosarcoma.

Findings: JZL184 reduced osteolytic bone metastasis in mouse models of breast and prostate cancers, and inhibited skeletal tumour growth, metastasis and the formation of ectopic bone in models of osteosarcoma. Additionally, JZL184 suppressed cachexia and prolonged survival in mice injected with metastatic osteosarcoma and osteotropic cancer cells. Functional and histological analysis revealed that the osteoprotective action of JZL184 in cancer models is predominately due to inhibition of tumour growth and metastasis. In the absence of cancer, however, exposure to JZL184 exerts a paradoxical reduction of bone volume via an effect that is mediated by both $\mathrm{Cnr} 1$ and $\mathrm{Cnr} 2$ cannabinoid receptors.

Interpretation: MAGL inhibitors such as JZL184, or its novel analogues, may be of value in the treatment of bone disease caused by primary bone cancer and bone metastasis, however, activation of the skeletal endocannabinoid system may limit their usefulness as osteoprotective agents.

(C) 2019 The Authors. Published by Elsevier B.V. This is an open access article under the CC BY-NC-ND license (http://
\end{abstract} creativecommons.org/licenses/by-nc-nd/4.0/).

\section{Introduction}

Monoacylglycerol lipase (MAGL) is a major enzyme of the endogenous cannabinoid (endocannabinoid) system that plays a role in neurotransmission, lipolysis and neuroinflammation [1-3]. MAGL is responsible for the degradation of the endocannabinoid 2-arachidonoyl glycerol (2AG) - the most abundant endocannabinoid, and acts as a

\footnotetext{
* Corresponding author at: Department of Oncology and Metabolism, Medical School, Beech Hill Road, Sheffield S10 2RX, UK.

E-mail address: aymen.idris@sheffield.ac.uk (A.I. Idris).

1 Authors contributed equally to the manuscript.
}

precursor to fatty acids $[1,3]$. Over recent years, a number of studies have shown that various types of tumours including breast and prostate carcinomas express MAGL [1,2,4], and its elevated level has been linked to malignancy, metastasis and poor patient prognosis and clinical outcomes [5-7]. In 2011, Nomura and colleagues uncovered the role of cancer-specific MAGL in prostate cancer progression, and showed that the verified MAGL inhibitor JZL184 reduced prostate cancer cell tumour growth and early metastasis by a mechanism that is dependent on levels of the 2AG and free fatty acids (FFA) [1,4].

The skeleton is a common site to metastatic cancer cells of various origin including prostate and breast, and the birthplace of bone sarcoma cells [8-14]. Disruption of the activity of immune and bone cells in 


\section{Research in context}

Evidence before this study

Cancer-induced bone disease is a serious complication in bone sarcomas and metastatic carcinomas of breast and prostate origin. Cannabinoids and their receptors ( $\mathrm{Cnr}$ ) are implicated in cancer and bone remodelling, while monoacylglycerol lipase (MAGL), an enzyme responsible for the degradation of the most abundant endocannabinoid in bone 2-arachidonoyl glycerol (2AG), has been shown to regulate tumour growth and early metastasis.

\section{Added value of this study}

Using mouse models of advanced breast and prostate cancer and osteosarcoma, we report that the verified MAGL inhibitor JZL184 inhibited cancer-related bone damage, reduced skeletal tumour growth, suppressed cachexia and prolonged survival. In the absence of cancer, however, exposure to JZL184 exerts a paradoxical reduction of bone volume via an effect that is mediated by cannabinoid receptors.

\section{Implications of all the available evidence}

In this study, we provide new insight in the role of the MAGL/2AG/ $\mathrm{Cnr}$ axis in normal and cancer-related bone remodelling, and conclude that targeting MAGL is of potential therapeutic efficacy in primary bone cancer and bone metastasis. However, we caution that activation of the skeletal endocannabinoid system in absence of cancer may limit the usefulness of MAGL inhibitors as osteoprotective agents.

particular the bone resorbing osteoclasts by cancer cells is a major contributory factor to the devolvement and progression of cancer associated bone disease $[8-10,15,16]$. Thus, treatments aimed at halting metastasis, reducing skeletal tumour growth, and attenuating osteoclastic bone damage would prove to be beneficial in terms of clinical outcomes in advanced cancer patients. Whilst the role of MAGL in cancer associated bone disease is unknown, the MAGL metabolite 2AG and its cannabinoid receptors ( $\mathrm{Cnr}$ ) have been previously implicated in the regulation of bone remodelling in health and disease [17-19].

The endocannabinoid 2AG is secreted in the skeleton by osteoblasts, osteoclasts and immune cells at levels similar to those present in the brain [19-25]. 2AG binds to cannabinoid receptors (Cnr) type 1 and 2 with varying degree of selectivity, and both Cnr1 and 2 have been found to be expressed by bone marrow, osteoblasts, osteoclasts and immune cells $[3,26,27]$. Skeletal analysis of transgenic animals showed that mice lacking Cnr1 or Cnr2 receptors develop osteoporosis with increasing age due to increased bone turnover [22,28-32], whereas genetic inactivation of both receptors in mice reduced age-related bone loss due to a reduction in osteoclast number [33]. Together, these findings consolidate the role of the endocannabinoid system in the regulation of bone remodelling.

Encouraged by the findings of Nomura et al. that implicated the 2AG/ MAGL axis in the prostate cancer initiation and progression [4], and the propensity of prostate cancer to metastasise to the skeleton [8-11,13], we tested the effects of MAGL knockdown and pharmacological inhibition on skeletal tumour burden, bone metastasis and osteolytic bone damage in preclinical models of bone disease associated cancers of prostate, breast and skeletal origin. Here, we report that MAGL inhibition reduced the initiation and progression of cancer related bone disease in models of secondary prostate and breast cancer in bone and osteosarcoma by a mechanism dependent - at least in part - on reduction of skeletal tumour growth and inhibition of osteolytic metastasis. In the absence of cancer, however, we show that exposure of host cells to the verified MAGL inhibitor JZL184 enhanced osteoclast formation and caused bone loss via a cannabinoid receptor dependent effect. Collectively, the results of the present finding implies that MAGL inhibitors such as JZL184, or its novel analogues, may be of value in the reduction of skeletal tumour burden and osteolysis caused by primary bone cancer and bone metastasis, however, activation of the skeletal endocannabinoid system may limit their usefulness as osteoprotective agents.

\section{Materials and methods}

\subsection{Reagents and chemicals}

The endocannabinoid 2AG and MAGL inhibitor JZL184 were purchased from Tocris Biosciences (Bristol, UK). The parental human breast MDA-MB-231, prostate PC3, osteosarcoma KHOS and Saos-2 were purchased from ATCC (Manassas, VA). The mouse osteosarcoma cells MOSJ were originally purchased from ATCC (Manassas, VA) and were a kind gift from Professor Dominique Heymann (INSERM, University of Nantes, France). The osteotropic human breast cancer cell line MDA-MB-231-BT (MDA-231-BT) was generated by Dr. Nadia Rucci (University of L'Aquila, Italy) and the osteotropic human prostate cancer cell line PC3-BT was a kind gift by Dr. Ning Wang (University of Sheffield, UK). Single cell clones of MDA-MB-231 were expanded and injected into the left ventricle of 4 -week-old female BALB/c-nu/nu mice. A clone that generated bone-only lesions with significant osteolysis in 8weeks was used [34-37]. Tissue culture medium (D-Modified Eagle Medium (MEM) and alpha-MEM) was obtained from Sigma-Aldrich (Dorset, UK). All primary antibodies were purchased from Cell Signalling Biotechnology (MA, USA) except rabbit anti-actin, which was obtained from Sigma-Aldrich (Dorset, UK). Mouse macrophage colony stimulating factor (M-CSF) was obtained from R\&D Systems (Abingdon, UK) and receptor activator of NFKB ligand (RANKL) was a gift from Patrick Mollat (Galapagos SASU, France).

\subsection{Animal experiments}

All procedures involving mice and their care were approved by and performed in compliance with the guidelines of Institutional Animal Care and Use Committee of Universities of Edinburgh (Scotland, UK), L'Aquila (L'Aquila, Italy) and Nantes (Nantes, France), and received institutional approval and conducted in conformity with laws and policies (UK Home Office and Italian and French authorities). C57BL/6 J and BALB/c-nu/nu mice were obtained from Harlan (UK) and Charles River (Milan, Italy), respectively. Six week-old female CD1 mice deficient in Cnr1 and Cnr2 receptors were generated as described in ${ }^{33}$.

\subsubsection{Intracardiac injection of human MDA-231-BT and PC3-BT tumour cells}

Eight week-old female BALB/c-nu/nu athymic mice were anesthetized with intraperitoneal injections of pentobarbital $(60 \mathrm{mg} / \mathrm{kg})$. Cancer cells re-suspended in PBS and intra-cardiacly injected $\left(2 \times 10^{6}\right.$ cells) ( 8 mice per group). Animals were divided into two/three groups and received intraperitoneal injection of either vehicle (water) or JZL184 (8-16 mg/kg). Animals were daily monitored for cachexia (evaluated by body weight waste), behaviour, and survival. At the end of the experiments, mice were euthanized by $\mathrm{CO}_{2}$ inhalation. Development of metastasis in bone was monitored by Bioluminescence imaging (PC3) using a IVIS SpectrumCT In Vivo Imaging System (PerkinElmer, UK) and X-ray analysis (PC3-BT and MDA-231-BT)(36 KPV for $10 \mathrm{~s}$ ) using a Cabinet X-ray system (Faxitron model n.43855A; Faxitron X-Ray Corp., Buffalo Grove, IL, USA) and Radiographs were scanned using the Bio-Rad scanning densitometer (Hercules, CA, USA), model GS800, and quantification of the area of interest was done using the Bio-Rad 
Quantity One image analysis software. At the end of the experiment (55 days from tumour-cell injection), mice were euthanized and subjected to micro-computed tomography (microCT) analysis and to anatomical dissection for evaluation of bone and visceral metastases, respectively. Skeletal tumour growth was measured on 2D microCT images using Image J (1.34 s; NIH, Bethesda, MD, USA) and results were expressed as a percentage of total metaphyseal area.

\subsubsection{Para-tibial injection of human KHOS and mouse MOS-J osteosarcoma cells}

Sixteen female NMRI athymic nude mice 5-week-old mice received para-tibial injection of human MNNG/HOS osteosarcoma cancer cells (2 $\times 10^{6}$ cells) and 16 female C57BL/6j 6-week old mice received paratibial injection of mouse MOSJ osteosarcoma cancer cells $\left(2 \times 10^{6}\right.$ cells) or a sham injection of PBS into their right leg. Animals were divided into two groups $(n=8)$ and received intraperitoneal injection of either vehicle (water) or JZL184 $(16 \mathrm{mg} / \mathrm{kg}$ ). Animals were euthanized 35 days post injection and bones were analyzed by microCT (Skyscan 1172 scanner). Lung metastasis and osteolysis were assessed as described above.

\subsection{Histomorphometry}

Metastatic bones were fixed in buffered 4\% formaldehyde and decalcified for 3 weeks. Bone histomorphometry was performed on the proximal tibial metaphysis from mice as previously described [38]. In short, bones were fixed, sectioned using a Leica microtome (Solms, Germany) and tissue sections KHOS model were stained with tartrateresistant acid phosphatase (TRACP) and haematoxylin and eosin (H\&E). Histomorphometry was performed on trabecular bone distal from the growth plate $(0.1$ and $1 \mathrm{~mm}$ distal to the growth plate). Bone histomorphometrical analysis was performed as previously described. Metastatic bones from the PC3-BT and MDA-231-BT were stained with H\&E and osteoclasts and osteoblasts were identified by morphology and contact with the bone. Histomorphometric measurements were carried out on $4 \mu \mathrm{m}$ thick sections, with an interactive image analysis system (Osteomeasure, Osteometrics Inc. Atlanta). Nomenclature, symbols and units of morphometric bone variables were used as previously described [39].

\subsection{Generation of stable cell lines}

To generate MAGL-shRNA expressing lentivirus 293 T-packaging cells $\left(1 \times 10^{5} \mathrm{cell} / \mathrm{cm}^{2}\right)$ were transfected with the $5 \mu \mathrm{g}$ pLKO.1 lentiviral vector (empty or containing MAGLshRNA), $5 \mu \mathrm{g}$ psPAX2 vector, $5 \mu \mathrm{g}$ pCMV-VSV-G vector and $40 \mu$ polyethylenimine. Twenty-four hours post-transfection, the medium was replaced with fresh standard D-MEM and cells were grown for an additional $24 \mathrm{~h}$. The conditioned medium containing recombinant lentiviruses was collected and filtered through $0.45 \mu \mathrm{m}$-pore-size filters. Samples of these supernatants were applied immediately to MDA-231-BT, PC3-BT or KHOS cells, which had been plated $18 \mathrm{~h}$ before infection at a density of $1 \times 10^{4}$ cells $/ \mathrm{cm}^{2}$. Polybrene (107,689 Sigma-Aldrich Dorset, UK) was added to a final concentration of $5 \mu \mathrm{g} / \mathrm{ml}$, and the lentiviral supernatants were incubated with the recipient cells. Twenty-four hours after infections, cells were selected with $1 \mu \mathrm{g} / \mathrm{ml}$ puromycin for $72 \mathrm{~h}$.

\subsection{Cancer cell lines and conditioned medium}

The osteotropic human MDA-231-BT, human PC3-BT and human KHOS cells were cultured in standard DMEM and mouse MOS-J cells were cultured in alpha-MEM (supplemented with $10 \%$ FCS, penicillin and streptomycin). For studies involving conditioned medium, cancer cells were allowed to grow to $80 \%$ confluence and the medium was refreshed with serum free D-MEM, and then conditioned medium was removed after $16 \mathrm{~h}$ and filtered (0.22 $\mu \mathrm{M}$ filter diameter). Freshly prepared conditioned medium ( $20 \% \mathrm{v} / \mathrm{v}$ ) was added to osteoclast and osteoblast cultures and their precursors in standard alpha-MEM supplemented. Cultures were then treated with vehicle or test compounds for the desired period of time.

\subsection{Osteoblast cultures}

Primary osteoblasts were isolated from the calvarial bones of 2-dayold mice by sequential collagenase digestion [40]. For bone nodule assay, osteoblasts $\left(10 \times 10^{5}\right.$ cells/12-well) were cultured in standard alpha-MEM, supplemented with $10 \%$ FCS, penicillin and streptomycin, $\beta$-glycerol phosphate $(10 \mu \mathrm{M})$ and L-ascorbic acid $(50 \mu \mathrm{g} / \mathrm{ml})$ for up to 21 days with replacement of the culture medium in the presence or absence of test substances every $48 \mathrm{~h}$. For osteoblast - cancer cell CM system, osteoblasts $\left(10 \times 10^{4}\right.$ cells/24-well) were cultured in alpha-MEM, supplemented with $10 \%$ FCS and 1,25-(OH)2-vitamin D3 (10nM) for up to 5-7 days with replacement of the culture medium in the presence or absence of test substances every $48 \mathrm{~h}$. Osteoblast number, differentiation and bone nodule formation were determined by AlamarBlue, alkaline phosphatase (Alk Phos) and alizarin red (ALZ) assays, respectively $[40,41]$.

\subsection{Osteoclast cultures}

Osteoclast formation, survival and activity were studied using RANKL and M-CSF generated mouse osteoclasts and bone-marrow cell - osteoblast co-cultures as previously described in [42]. Briefly, bone marrow (BM) cells were flushed from the long bones of 3-5 month old mice and were cultured in standard alpha-MEM supplemented with mouse M-CSF ( $100 \mathrm{ng} / \mathrm{ml}$ ). Erythrocytes were removed and adherent cells were re-suspended in culture medium and counted. For osteoclast generation, M-CSF-generated bone marrow macrophages (preosteoclasts) were plated into tissue culture plates (96-well plates, 15 $\times 10^{3}$ cells/well; 12 -well plates, $150 \times 10^{3}$ cells/well) in standard alpha-MEM supplemented with M-CSF (25 ng/ml) and RANKL (100 ng/ml). The macrophage cell line RAW 264.7 was cultured in 96well plates $\left(15 \times 10^{3}\right.$ cells/well $)$ in standard alpha-MEM supplemented with RANKL (100 ng/ml). For studies involving conditioned medium, conditioned medium from cancer cultures was prepared as described above then added to osteoclast cultures at a concentration of $10 \%$ $(v / v)$ in standard alpha-MEM. The cultures were terminated by fixation in $4 \%$ paraformaldehyde and washed with PBS. TRAcP staining was used to identify multi-nucleated osteoclasts and TRAcP positive cells with 3 or more nuclei were considered to be osteoclasts [43].

\subsection{Assessment of cell motility}

Cell migration was assessed by wound healing assay. Briefly, cells were plated into 24 -well plates $\left(2-5 \times 10^{5}\right.$ cells/well $)$ and confluent cell layers were scored with a fine pipette tip to produce a wound. Migration of cells across the wound was monitored using an Olympus ScanR time lapse microscope system and percentage of wound closure were analyzed using the ImageJ program. Cell spreading was assessed by using the real-time cell analyzer xCELLigence (Roche Applied Science, UK) $[41,44]$. Briefly, cells $\left(10^{3}\right.$ cells/well) were plated into specialized 16-well plates (Roche Applied Science, UK) in alpha-MEM in the presence and absence of JZL184 for 2-6 h. Data were analyzed using the xCELLigence software [44].

\subsection{Micro-computed tomography}

Ectopic bone formation (tibia and fibula) and trabecular bone parameters (tibia and femur) were measured using micro-computed tomography (microCT) analysis (Skyscan 1076 Brucker, Belgium) set at $50 \mathrm{kV}$ and $200 \mathrm{~mA}$. Skyscan NRecon sofware was used to reconstruct 
and images were analyzed using CTan (Skyscan CTAn, Brucker, Belgium) [45].

\subsection{LC-MS/MS}

Liquid chromatography-mass spectrometry (LC-MS/MS) was used to measure the levels of endocannabinoids as previously described [23]. Briefly, cell pellets or conditioned medium were re-suspended in $600 \mu \mathrm{l}$ of Lysis Buffer ( $10 \mathrm{mM}$ Tris, $1 \mathrm{mM}$ EDTA, $\mathrm{pH}=7.6$ ) and mechanically disrupted using a polytron device ( $20 \mathrm{Krpm}, 30 \mathrm{~s}$, in ice) and by one-cycle of freeze thawing. Cell lysates were cleared up by centrifugation (4000 rpm, $5 \mathrm{~min}, 4^{\circ} \mathrm{C}$ ) and the supernatants were separated and stored at $-80^{\circ} \mathrm{C}$. Total protein was determined using the BCA kit (Bio Rad).

\subsection{Western blotting}

Western blot analysis was used to detect protein expression. Cells were lysed in a standard buffer $(0.1 \%(\mathrm{w} / \mathrm{v})$ Sodium dodecyl sulfate, $0.5 \%(\mathrm{w} / \mathrm{v})$ sodium deoxycholate, $1 \%$ Triton X-100, $1 \mu \mathrm{M}$ Ethylenediaminetetraacetic acid, $2 \%(\mathrm{v} / \mathrm{v})$ protease inhibitor cocktail, $10 \mu \mathrm{M}$ of sodium fluoride and $2 \%(\mathrm{v} / \mathrm{v})$ phosphatase inhibitor cocktail. Protein concentration was determined using BCA assay (Pierce, USA). Total protein $(60 \mu \mathrm{g})$ was resolved by SDS-PAGE (BioRAD, United Kingdom), immunoblotted with antibodies, detected using rabbit monoclonal antibodies (all at 1:1000 dilution, cell Signalling Technology, USA) and immuno-complexes were visualised by an enhanced chemiluminescence (Amersham, UK) on a Syngene GeneGnome imaging system. The intensity of the bands was quantified using GeneSnap software (Syngene, UK) and level of actin was used for normalization.

\subsection{Measurement of levels of tumour-derived factors}

Level of tumour-derived factors in conditioned medium was measured by using the Proteome Profiler Human XL Cytokine Array Kit (ARY022, R\&D Systems, Abingdon, UK), according to the manufacturer's instructions.

\subsection{Statistical analysis}

Data analysis and statistical comparisons between groups were made by use of Student's $t$-test or analysis of variance (ANOVA) followed by Dunnet's post test (SPSS for Windows, version 11). A pvalue value of 0.05 or below was considered statistically significant.

\section{Results}

\subsection{MAGL inhibition reduces migratory and osteolytic features of osteotropic cancer cells}

MAGL is implicated in cancer progression and the administration of JZL184 in mice reduced tumour growth and overt metastasis in the human prostate cancer PC3 model [1,4]. Metastatic cancer cells of prostate origin including PC3 cells preferably metastasise to bone [8-12], however the effect of MAGL inhibition on bone metastasis has not been investigated. Here, we first confirmed that MAGL is expressed in patients (Supplementary Fig. S1A-D) and mice (Supplementary Fig. S1E) with prostate cancer, and its expression correlates with the metastatic potential of osteotropic PC3 cells when compared to poorly metastatic LNCap cells (Supplementary Fig. S1F). MAGL is expressed in biopsies from osteosarcoma patients (Supplementary Fig. S2A-B), and its activity was detected and successfully knocked down in the human osteotropic sub-clones of prostate PC3 (PC3-BT) and breast MDA-MB-231 (MDA-231-BT) (Supplementary Fig. S3). In view of these findings and the fact that cancer cells in the skeleton grow, move, alter the behaviour of bone cells differently compared to their parental counterpart [8-10,15,46], we assessed the effect of JZL184 on the growth and metastatic behaviour of a panel of human and mouse osteotropic prostate and breast cancer and osteosarcoma cells (Fig. 1, Fig. S4, and data not shown). JZL184 $(1-100 \mu \mathrm{M})$ reduced the viability of human, osteotropic prostate PC3-BT and breast MDA-231-BT and the osteoblast-like osteosarcoma cell line cells Saos2 in a concentrationdependent manner after continuous exposure for $72 \mathrm{~h}$ (Fig. S4A). In contrast, JZL184 enhanced the viability of the naïve PC3, MDA-231 and exerted no significant effects on the growth of the highly differentiated and metastatic osteosarcoma cells KHOS at concentrations up to $100 \mu \mathrm{M}$ (Fig. S4A). Next, we knocked down the protein level of MAGL (Fig. S3A), and went on to show that both MAGL knockdown and exposure to JZL184 $(3 \mu \mathrm{M})$ reduced the directed migration (Fig. 1A), invasion (Fig. 1B) and growth (Fig. S4) of the osteotropic PC3 and MDA-231 and KHOS. Additionally, exposure of M-CSF pre-treated mouse bone marrow derived osteoclast precursors to JZL184 (1-3 $\mu \mathrm{M})$ prior to addition of mock or MAGL-deficient prostate PC3-BT, breast MDA-231-BT, or osteosarcoma KHOS cell lines significantly inhibited osteoclast formation (Fig. 1C), indicative of an anti-osteolytic effect (Fig. 1D). Together, these in vitro results suggest that JZL184 may be of benefit in reducing the ability of tumour cell to cause cancer-associated bone disease in mouse models of the human breast, prostate and osteosarcoma cell lines described (Fig. S4).

\subsection{JZL184 reduces skeletal tumour growth, metastasis and cachexia and improves survival}

Next, we inoculated mice with the human breast MDA-231-BT and prostate PC3-BT and osteosarcoma KHOS and the syngeneic osteosarcoma MOS-J cells, and tested the effects of JZL184 on the initiation and development of cancer associated bone disease (Fig. 2A and F). Graphic representation of intra-cardiac injection of osteotropic human prostate PC3-BT and breast MDA-231-BT and para-tibial injection of the human KHOS and mouse MOS-J into mice are shown in Figs. S5, S7 and S8, respectively. The dosing regime of JZL184 have been chosen on the basis of previous studies that have shown it produced cannabinoid behavioral effects by increasing the level of the endocannabinoid 2AG [47-50], and reduced tumour growth and metastasis $[4,51,52]$. The rationale for choosing JZL184 at $16 \mathrm{mg} / \mathrm{kg}$, thrice weekly in most of our studies is that previous studies have reported a near-complete blockade of MAGL by JZL184 at this dose [47-50]. Administration of JZL184 (16 mg/kg, thrice weekly) inhibited the skeletal tumour growth (Fig. 2A-C) of the human prostate PC3-BT, breast MDA-231-BT and osteosarcoma KHOS cells in immuno-deficient nude mice and mouse MOS-J cells in immuno-competent mice (Fig. 2A). Representative images from the osteotropic PC3-BT and osteosarcoma KHOS experiment described are shown in Fig. 2 panels B and C, respectively. Additionally, treatment with JZL184 (16 mg/kg, thrice weekly) reduced the incidence of bone metastasis of the human osteotropic prostate PC3-BT (Fig. 2D) and breast MDA-231-BT cells (supplementary Fig. S5A-B), and inhibited the metastasis of the osteosarcoma human KHOS and mouse MOS-J cells to the lungs (Fig. 2E). JZL184 also reduced cachexia in mice after intra-cardiac injection of osteotropic MDA-231-BT cells (Fig. S5C), and improved survival in this model (Fig. S5D) and in mice locally injected with the osteosarcoma KHOS (Fig. 2F). In contrast, mice inoculated with MAGL deficient KHOS cells exhibited similar tumour growth (supplementary Fig. S6A) and non-significant reduction in number of lung micro-metastasis (supplementary Fig. S6B) when compared to mock control.

\subsection{JZL184 reduces cancer induced bone loss}

Detailed microCT analysis of the long bones of mice (Fig. 3A-C) showed that injection of the human prostate PC3-BT (Fig. 3A), breast MDA-231-BT (Fig. 3A) and osteosarcoma KHOS (Fig. 3A) cells in immuno-deficient nude mice caused significant osteolytic bone damage characterised by significant reduction in bone volume when compared 

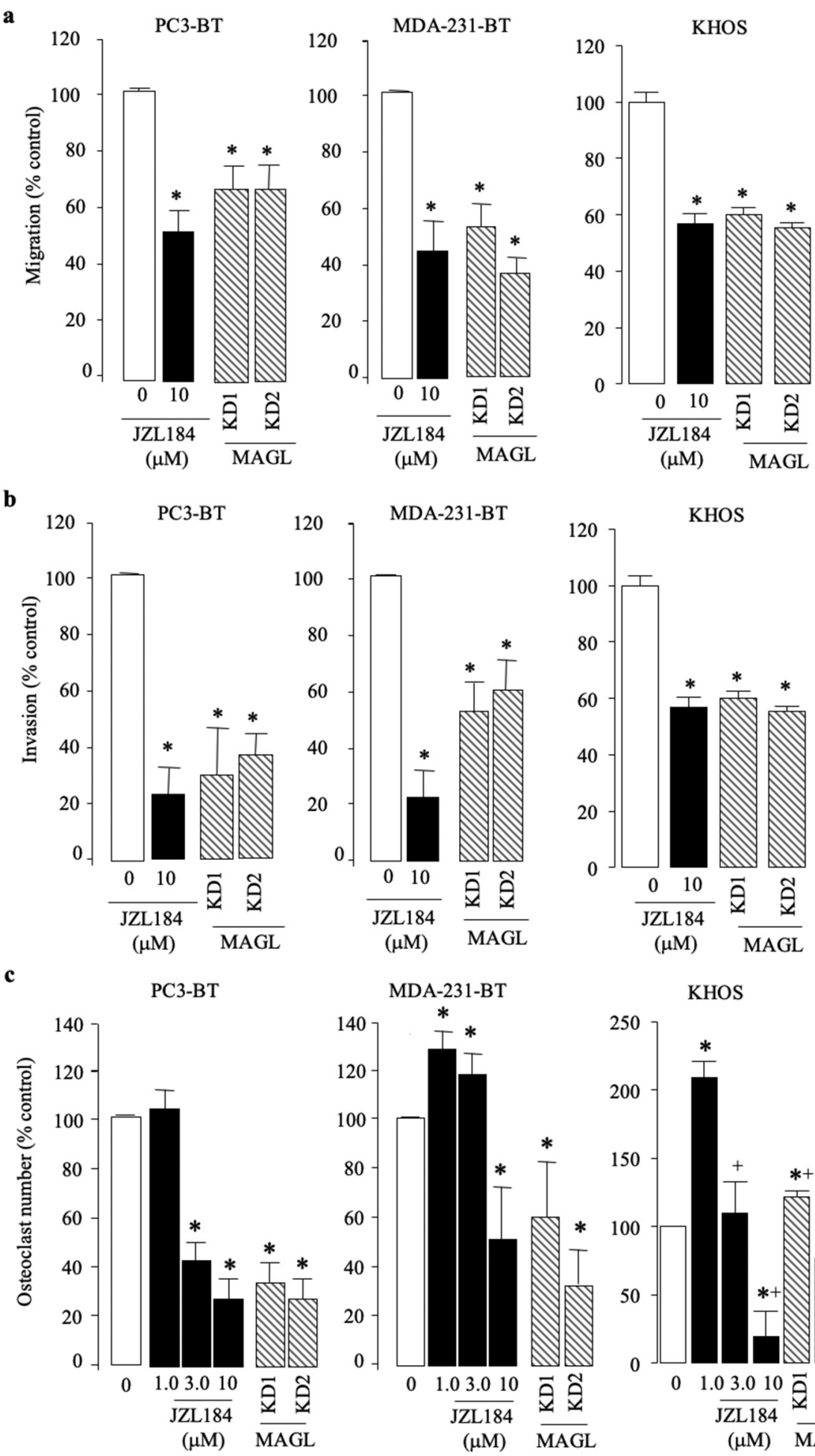

KHOS
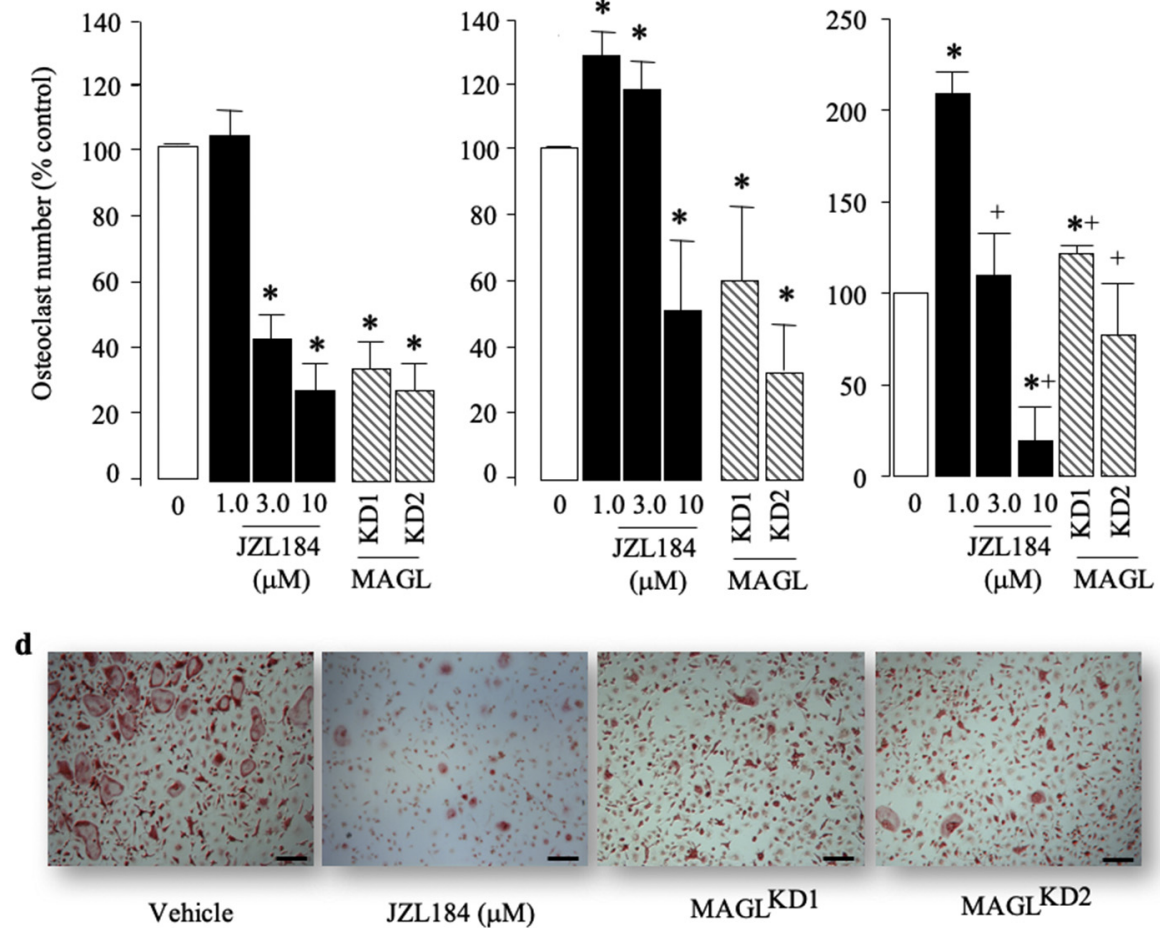

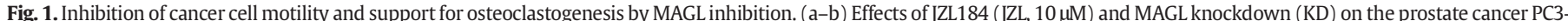

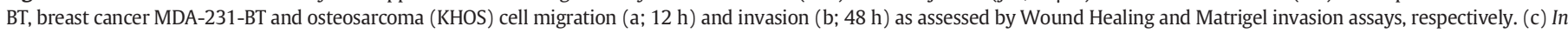

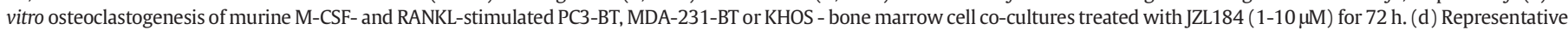

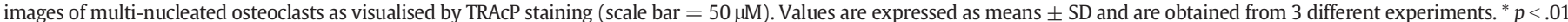
from control cultures and $+\mathrm{p}<.01$ between two cell types tested. 
to the sham leg and these effects were significantly reduced by administration of JZL184 (16 mg/kg, thrice weekly). In the osteolytic bone metastasis models, MDA-231-BT (supplementary Fig. S5E) and PC3-BT (supplementary Fig. S8D), JZL184 (16 mg/kg, thrice weekly) increased trabecular number (Tb.N), reduced trabecular separation (Tb.Sp) and increased trabecular connectivity (reduced trabecular pattern factor; $\mathrm{Tb}$. Pf). Representative microCT images from the MDA-231-BT experiment described are shown in Fig. 3 (panel D). In the MDA-231-BT model, JZL184 exerted similar osteoprotective effects in mice at doses of both $8 \mathrm{mg} / \mathrm{kg}$ and $16 \mathrm{mg} / \mathrm{kg}$ thrice weekly (supplementary Fig. S5E). Similarly, JZL184 (16 mg/kg, thrice weekly) increased bone volume (Fig. 3C) and trabecular connectivity (reduced trabecular pattern factor; Tb.Pf; Fig. S7C) in the KHOS mouse model. In this model, however, injection of the human osteosarcoma KHOS cells in these mice enhanced trabecular thickness and this effect was enhanced by JZL184 treatment (Fig. S7D). Histological and histomorphometrical analysis of bones revealed that JZL184 (16 mg/kg, thrice weekly) increased osteoclast (Fig. 3D) and reduced osteoblast (Fig. 3E) activity in healthy legs of mice that received para-tibial injection of human KHOS osteosarcoma cells but had no significant effects on both parameters in legs bearing osteosarcoma KHOS, prostate PC3-BT or breast MDA-231-BT tumours. In contrast, we observed non-significant increase in bone volume in mice inoculated with osteolytic MDA-231-BT (supplementary Fig. S6C) or mixed osteoblastic/ osteolytic KHOS cells (data not shown) deficient in MAGL when compared to mock control. Collectively, these results show that JZL184 reduces cancer induced bone damage in the mouse models of osteosarcoma, prostate and breast cancer described.

\subsection{JZL184 inhibits cancer-associated ectopic bone formation}

In view of the fact that osteosarcoma cells induce the formation of ectopic bone in human [53] and in the mouse models used in this study [54,55], and JZL184 inhibited osteoblast activity in the KHOS osteosarcoma model (Fig. 3F), we carried out further microCT analysis of cancer-induced bone formation within the tumour in close proximity to the fibula and proximal tibia of mice. As shown in Fig. 4, mice that received JZL184 (16 mg/kg, thrice weekly) exhibited reduced ectopic bone volume in both tibia (Fig. 4A) and fibula (Fig. 4C) when compared to vehicle treated mice. Representative photomicrographs of ectopic bone formation from the experiment described are shown in Fig. 4, panels B and D. Next, we carried out additional in vitro studies on the effects of JZL184 on the ability of calvarial mouse osteoblast and the human osteoblast-like and early-differentiated osteosarcoma cells Saos2 to form bone nodule in the presence and absence of derived factors from the highly metastatic KHOS osteosarcoma cells. These experiments showed that exposure to JZL184 $(1 \mu \mathrm{M})$ reduced alkaline phosphatase activity and bone nodule formation in cultures of mouse osteoblasts (Fig. 4E, left) and Saos2 (Fig. 4E, right). Similar inhibition in alkaline phosphatase activity and bone nodule formation was also observed in osteoblasts (Fig. 4G, left) and Saos2 (Fig. 4G, right) cultured in the presence of conditioned medium from KHOS osteosarcoma cells. Interestingly, exposure to JZL184 $(10 \mu \mathrm{M})$ had no significant effects on the viability of calvarial osteoblasts in the experiments described but a modest reduction in the viability of the Saos2 osteosarcoma cells was observed particularly in the presence of conditioned medium from KHOS osteosarcoma cells (Fig. 4G, right). Representative photomicrographs of bone nodule formation from the calvarial osteoblast cultures in the absence or presence of conditioned medium from KHOS osteosarcoma cells are shown in Fig. 4, panel $\mathrm{F}$ and $\mathrm{H}$, respectively. These results together indicate that exposure of osteoblasts and early-differentiated osteosarcoma cells to JZL184 reduce their ability to differentiate and form bone nodule.

\subsection{JZL184 causes paradoxical bone loss by cannabinoid receptor activation}

MAGL is responsible for the degradation of 2AG, the endocannabinoid which activates both $\mathrm{Cnr} 1$ and $\mathrm{Cnr} 2$ receptors
[19-25]. 2AG is produced within the central nervous system and in the bone microenvironment by osteoblasts and osteoclasts [19,21-24], and both cannabinoids receptors $\mathrm{Cnr} 1$ and $\mathrm{Cnr} 2$ have been implicated in the regulation of bone cell activity and bone remodelling $[22,28,30,32,33,56-58]$. In view of these findings and the present observation that administration of JZL184 showed a trend towards reduced cortical (Fig. S7B, left) and trabecular (Fig. S7B, right) bone volume in non-tumour bearing legs of mice, we carried out additional in vivo experiments to test the effect of JZL184 on bone mass in wild type (wt) and double Cnr1 and Cnr2 knockout ( $\left.\mathrm{Cnr} 1 / 2^{-/-}\right)$female mice treated. As shown in Fig. 5, administration of JZL184 (16 mg/kg, thrice weekly) in wild type mice for 60 days caused bone loss characterised by a significant reduction in bone volume (Fig. 5A) and trabecular connectivity (increase in trabecular separation, Fig. 5B) and number (Supplementary Fig. S9A). In contrast, Cnr1/2 $2^{-1-}$ female mice treated with JZL184 $(16 \mathrm{mg} / \mathrm{kg}$, thrice weekly) were completely protected and exhibited similar trabecular bone volume and connectivity comparable to vehicle treated control (Fig. 5A-B). Representative microCT images from the experiment described are shown in Fig. 5 (panel C). Interestingly, neither JZL184 treatment nor genetic inactivation of Cnr1 and Cnr2 receptors resulted in any significant changes in trabecular thickness (Supplementary Fig. S9B) or cortical bone parameters (data not shown). Histological and histomorphometrical analysis of bones revealed that JZL184 $(16 \mathrm{mg} / \mathrm{kg}$, thrice weekly) increased the activity of both osteoclasts (Supplementary Fig. S9C) and osteoblasts (Supplementary Fig. S9D), and these effects were significantly blunted in Cnr1/2 $2^{-/-}$mice. To investigate whether tumour-derived endocannabinoids and/or host-specific classical cannabinoid receptors $\mathrm{Cnr} 1$ and $\mathrm{Cnr} 2$ are implicated in the pro-osteolytic action of JZL184, we first used liquid chromatography-mass spectrometry (LC-MS/MS) to analyze the levels of endocannabinoids in conditioned medium from the osteolytic MDA231-BT cells. This experiment shows that MDA-231-BT cells produce the endocannabinoid 2AG (Fig. 5D) and its level is significantly increased by addition of the osteolytic factor, RANKL (Fig. 5E). Interestingly, MDA-231-BT-derived 2AG was detected in higher level than Anandamide (AEA) and the prostaglandin PGE2, but lower level than Arachidonic acid (AA) (Fig. 5D). Next, we used the mouse calvarial organ - human cancer cell co-culture model (Fig. 5F) to test if the effect of MAGL inhibition by JZL184 on cancer-induced osteolysis is mediated by activation of classical cannabinoid receptors expressed by host cells. The main advantage of the calvaria organ culture used in this study it allowed us to examine the effects of JZL184 on osteolytic bone damage induced by human cancer cells in skeletal tissue derived from both immuno-competent wild type and Cnr1/2 double knockout mice from the $\mathrm{CD} 1$ background described. These experiments showed that the addition of the osteolytic MDA-231-BT - but not prostate cancer PC3-BT or osteosarcoma KHOS (data not shown) - cells to the organ cultures from wild type female mice caused a decrease in calvarial bone volume $(p<$ .01 ) over a 7 day culture period and this effect was significantly reduced in organ cultures from Cnr1/2 double knockout female mice (Fig. 5G). Treatment with JZL184 $(1 \mu \mathrm{M})$ increased osteolysis even further and this effect was partially reversed in organ cultures from $\mathrm{Cnr} 1 / 2^{-1-}$ mice cultured with mock or MDA-231 deficient in MAGL (MAGL ${ }^{\mathrm{KD} 1}$ ) (Fig. 5G), indicative of the involvement of cancer-specific MAGL and host specific Cnr1 and 2 receptors. Representative microCT images from the experiment described are shown in Fig. 5H. To examine the contribution of individual cannabinoid receptors, we pre-treated the mouse calvarial - MDA-231-BT cancer cell co-cultures with the Cnr1 selective inverse-agonist AM251 or the Cnr2 selective inverse-agonist AM630 for $1 \mathrm{~h}$ prior to the addition of JZL184 for 5 days. As shown in supplementary Fig. S9, treatment with AM630 (3 $\mathrm{MM}$ ), and to lesser extent AM251, significantly blunted the pro-osteolytic action of JZL184 (1 $\mu \mathrm{M})$. It is important to note that exposure to AM251 and AM630 $(3 \mu \mathrm{M})$ significantly reduced the viability of MDA-231-BT cells under conditions tested by $27 \pm 12 \%$ and $37 \pm 4 \%(p<.05)$, respectively. Interestingly, pre-treatment of the MDA-231-calvarial bone co-culture with the 


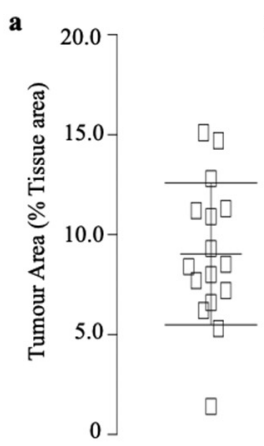

PC3-BT

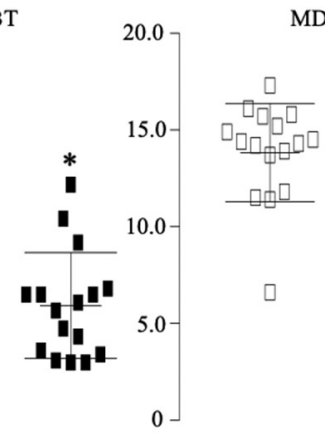

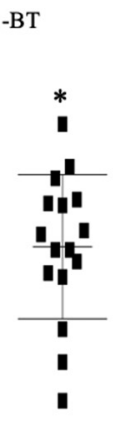

PC3-BT
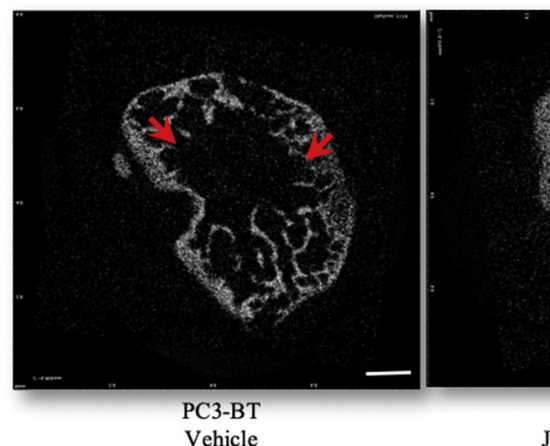

e

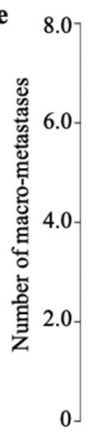

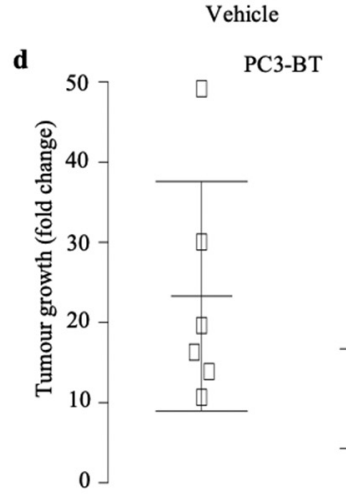

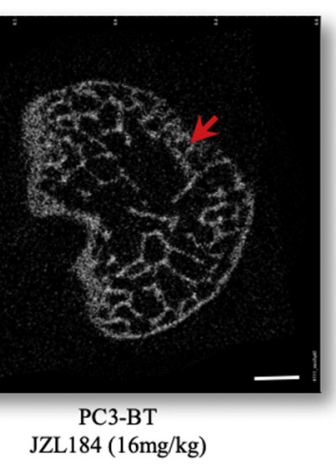

KHOS

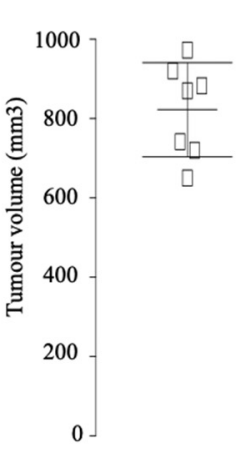

KHOS

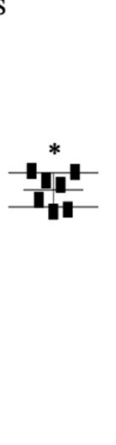

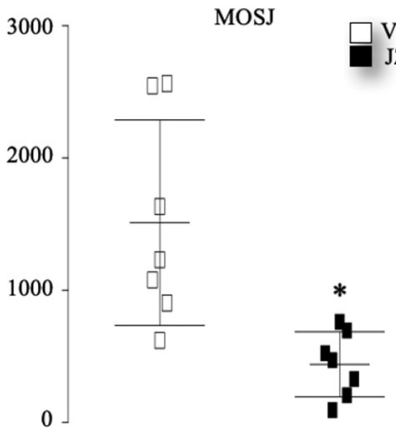

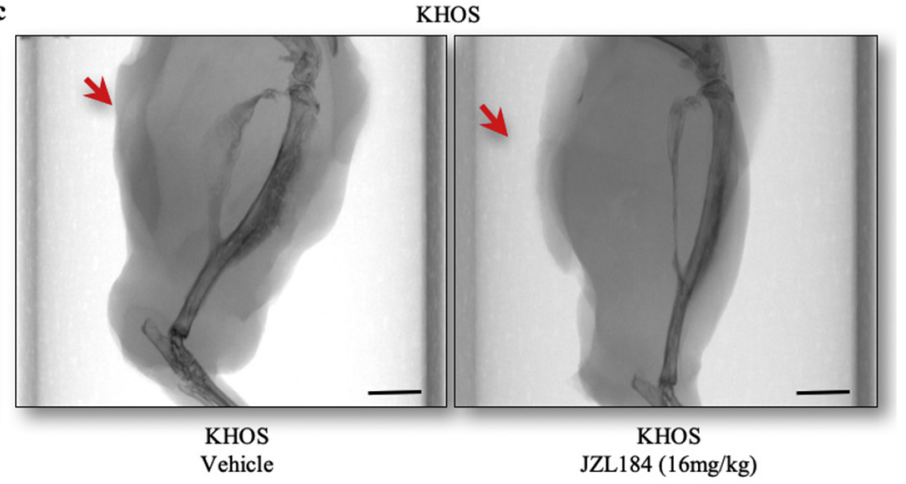

KHOS

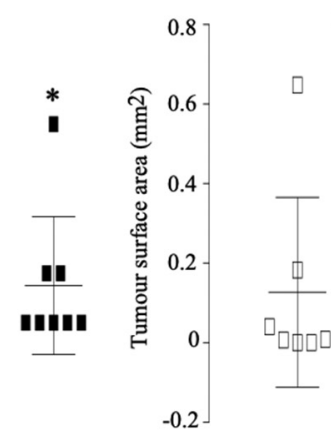

MOSJ
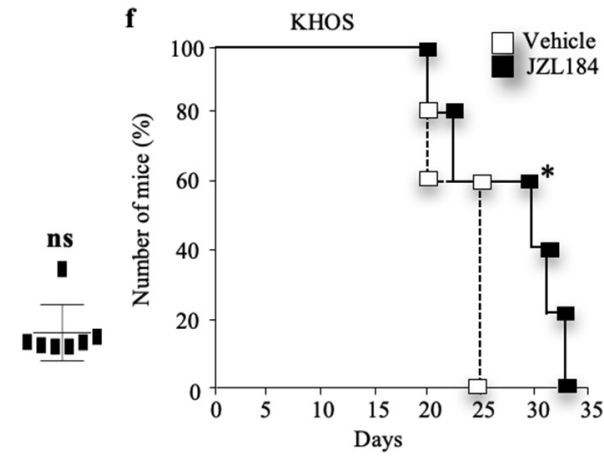

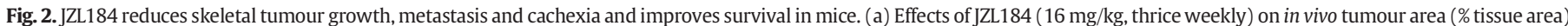

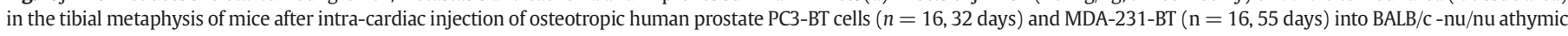

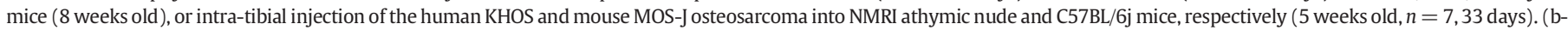

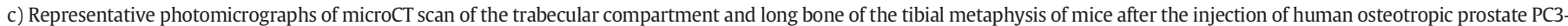

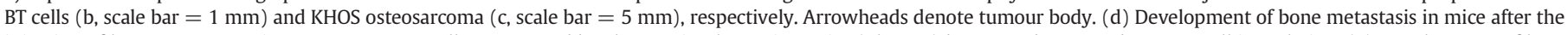

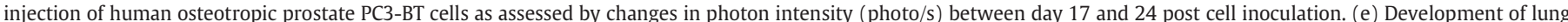

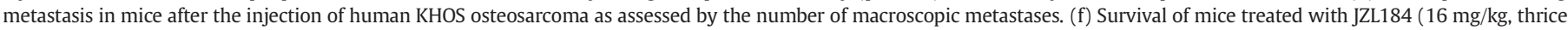
weekly) for 33 days after para-tibial injections of the human KHOS cells $(n=7-8)$ from the experiment described in panels F-H. Values are mean \pm SD; ${ }^{*} p<.05$.

cox1/2 inhibitor Indomethacin $(10 \mu \mathrm{M})$ failed to protect against JZL184 $(3 \mu \mathrm{M})$ driven osteolysis (Fig. 5I), excluding the involvement of the prostaglandin/Cox pathway in the model described.

\subsection{JZL184 disrupts cancer cell - osteoclast crosstalk}

Analysis of the levels of human cytokines and chemokines in conditioned medium from MDA-231-BT revealed that exposure to JZL184 at 1 $\mu \mathrm{M}$ - the concentration that enhanced MDA-231-BT-induced osteolysis in the calvarial model - increased the levels of MDA-231-BT-derived vascular endothelial growth factor (VEGF), resistin and to a lesser extend brain-derived neurotrophic factor (BDNF) and Granulocytemacrophage colony-stimulating factor (GM-CSF) (Fig. 5J) - four factors that are known to enhance osteoclast formation [59-63]. JZL184 (1 $\mu \mathrm{M}$ ) also increased the level of sex hormone-binding globulin (SHBG), a serum glycoprotein that has been associated with bone loss and the occurrence of fractures. In contrast, we detected a reduction ( $\leq 1 \mathrm{fold} / \mathrm{ve}-$ hicle) in the osteoclast inhibitory factors B-cell-activating factor (BAFF)
[64], macrophage Migration Inhibitory Factor (MIF) [65], trefoil factor family 3 (TFF3) [66], osteopontin (OPN) [67] and myeloperoxidase (MPO) [68]. Interestingly, JZL184 $(1 \mu \mathrm{M})$ reduced the level of the proinflammatory TNF $\alpha$, which is known to both enhance [69] and reduce [70] osteoclast formation, and induces cell death and apoptosis in cells of the osteoblast lineage [69,71]. We also carried out quantitative PCR analysis of MAGL expression in bone marrow cells (BMCs), osteoblasts, osteoclasts and their precursor cells. As shown in Fig. 6A, mRNA levels of MAGL in bone marrow cells and calvarial osteoblasts were significantly higher than in mature osteoclasts and their M-CSF dependent bone marrow macrophage (osteoclast precursors). Together, these results suggest that JZL184 disrupts cancer cell - osteoclast crosstalk in osteolytic environment.

\subsection{JZL184 and 2AG exert a biphasic effects on osteoclastogenesis}

To investigate the effects of JZL184 on cancer-induced osteoclast formation further, we went on to show that pre-exposure of mouse bone 

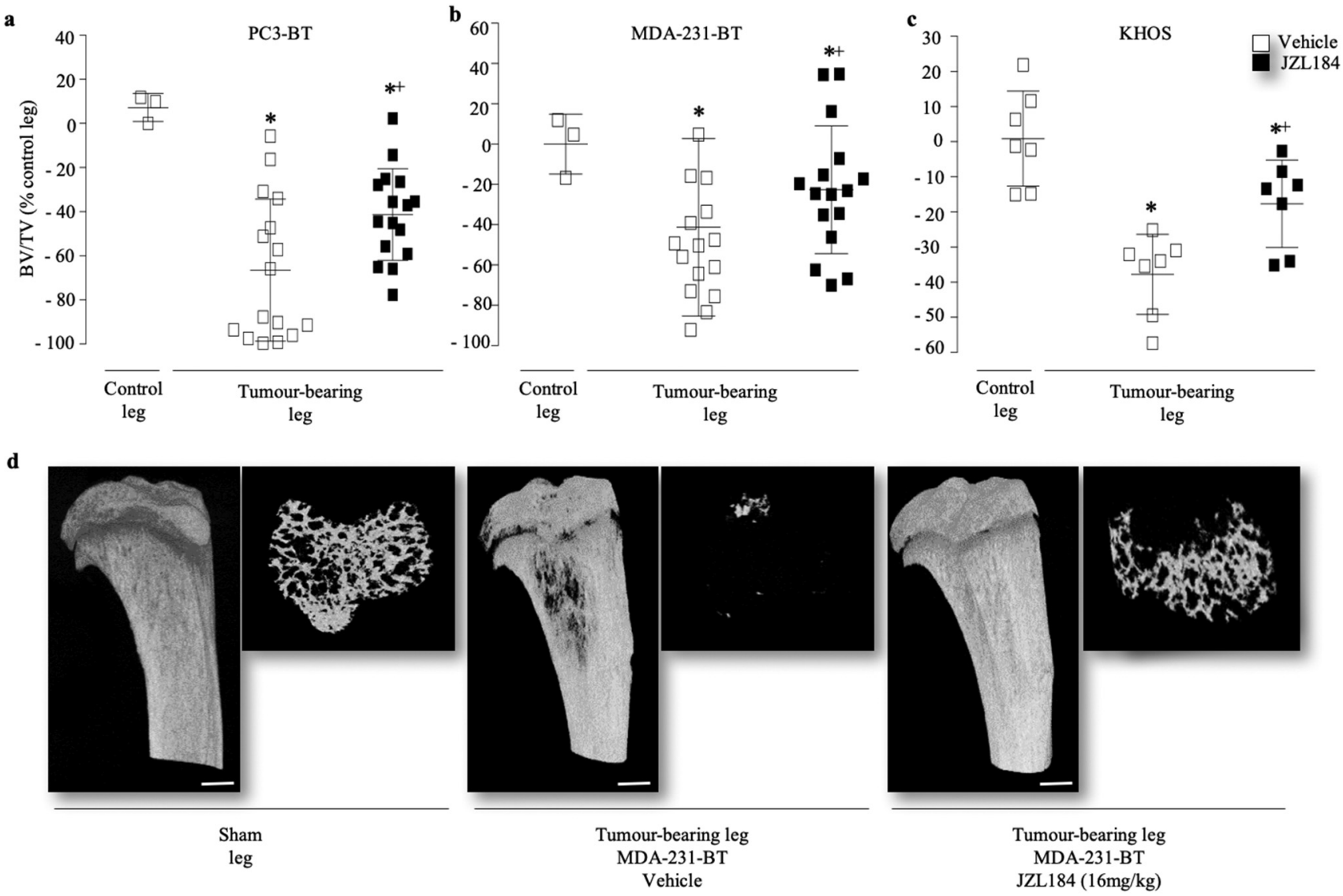

e

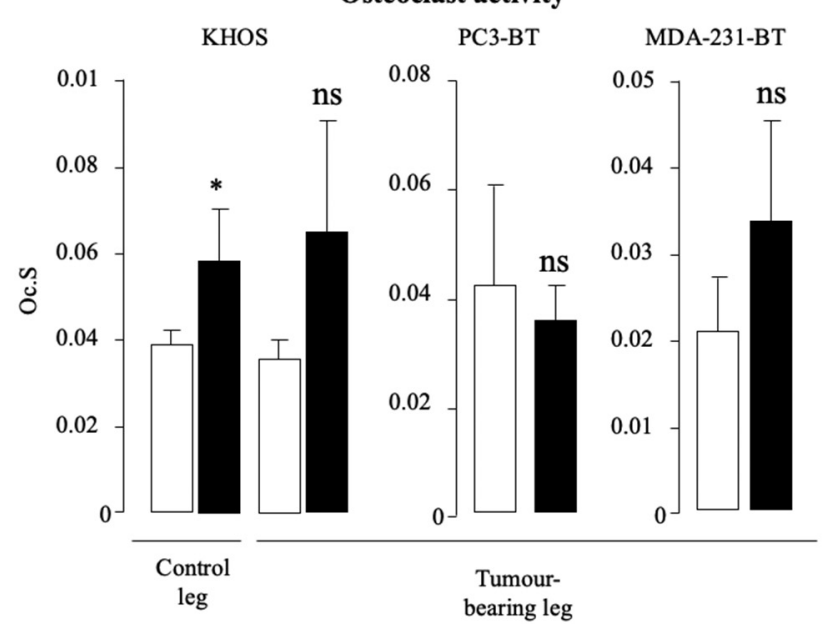

f

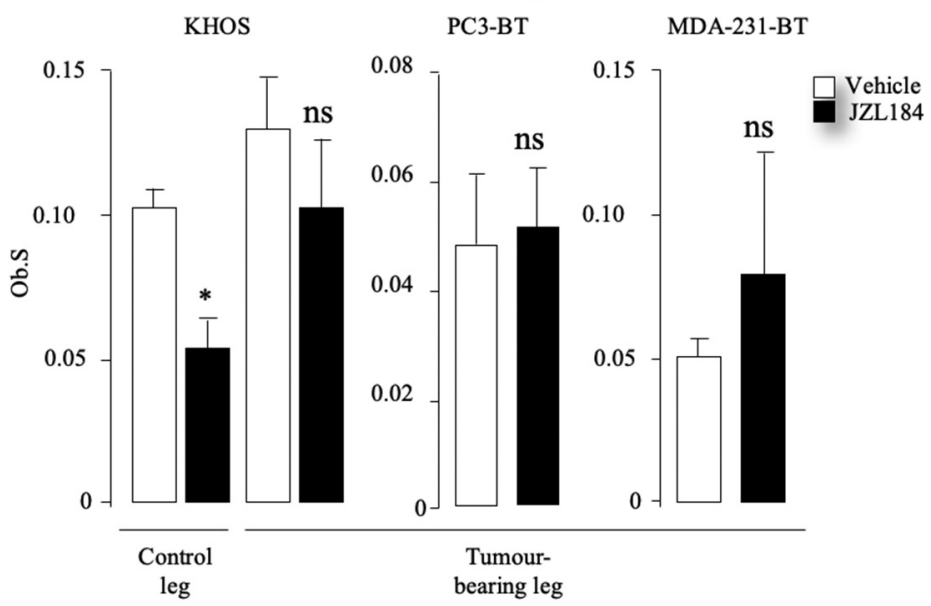

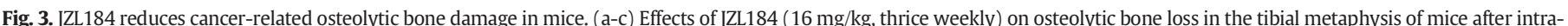

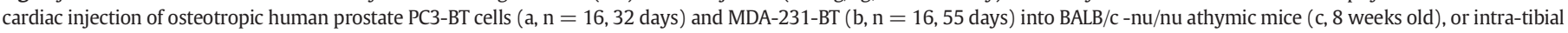

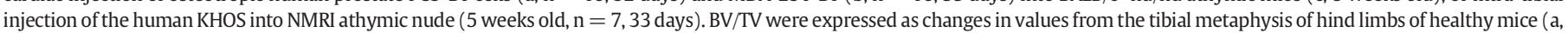

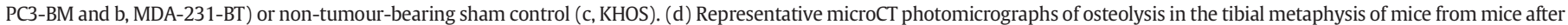

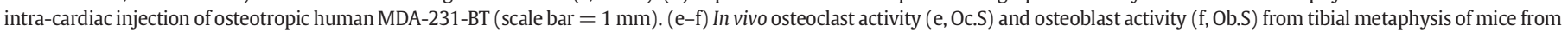
the experiment described in panels a - d, as assessed by histomorphometrical analysis. Values are mean $\pm \mathrm{SD} ;{ }^{*} p<.05$. ns denotes not statistically significant.

marrow cells (pre-osteoclasts) to JZL184 ( 0.1 and $0.3 \mu \mathrm{M})$ in the absence (Fig. 6B, left) or presence of calvarial osteoblasts (Fig. 6B, middle) or a cocktail of RANKL (50 ng/ml) and M-CSF (25 ng/ml) (Fig. 6B, right) increased the formation of TRAcP positive osteoclasts. At $10 \mu \mathrm{M}$, however, JZL184 significantly reduced osteoclast formation in BMC-osteoblast cocultures (Fig. 6B, middle) and in the presence RANKL and M-CSF (Fig. 5B, right). Although M-CSF dependent bone marrow macrophage expressed a low level of MAGL mRNA (Fig. 6A), JZL184 (1 - $10 \mu \mathrm{M})$ exerted similar biphasic effects on the ability of these cells to grow (Fig. 6C, left), spread (Fig. 6C, middle) and form mature osteoclasts in the presence of RANKL (Fig. 6C, right). We also investigated whether $2 \mathrm{AG}$, the principal endocannabinoid metabolized by MAGL, influence osteoclastogenesis in the model described. As shown in Fig. 5C, exposure of M-CSF dependent bone marrow macrophage to 2AG (0.1 - 10 $\mu \mathrm{M})$ enhanced the action of RANKL (100 ng/ml) and M-CSF $(25 \mathrm{ng} / \mathrm{ml})$ on the expression of a panel of osteoclast specific genes 

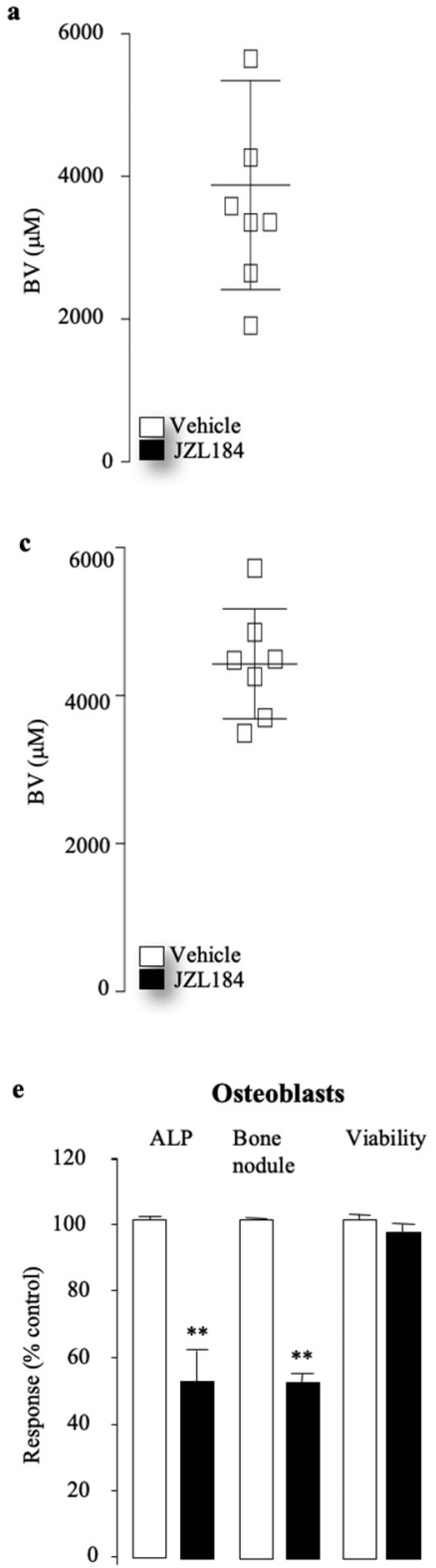

g

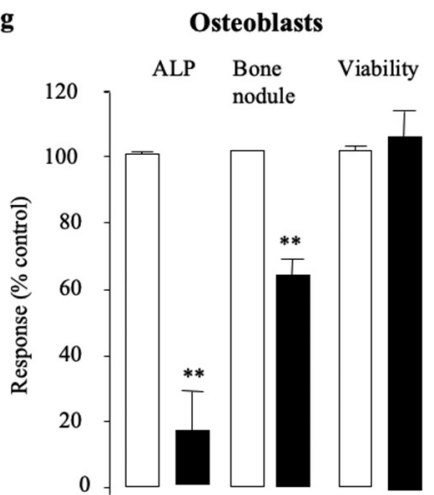

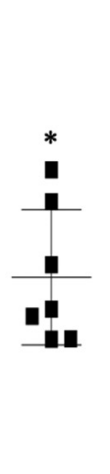
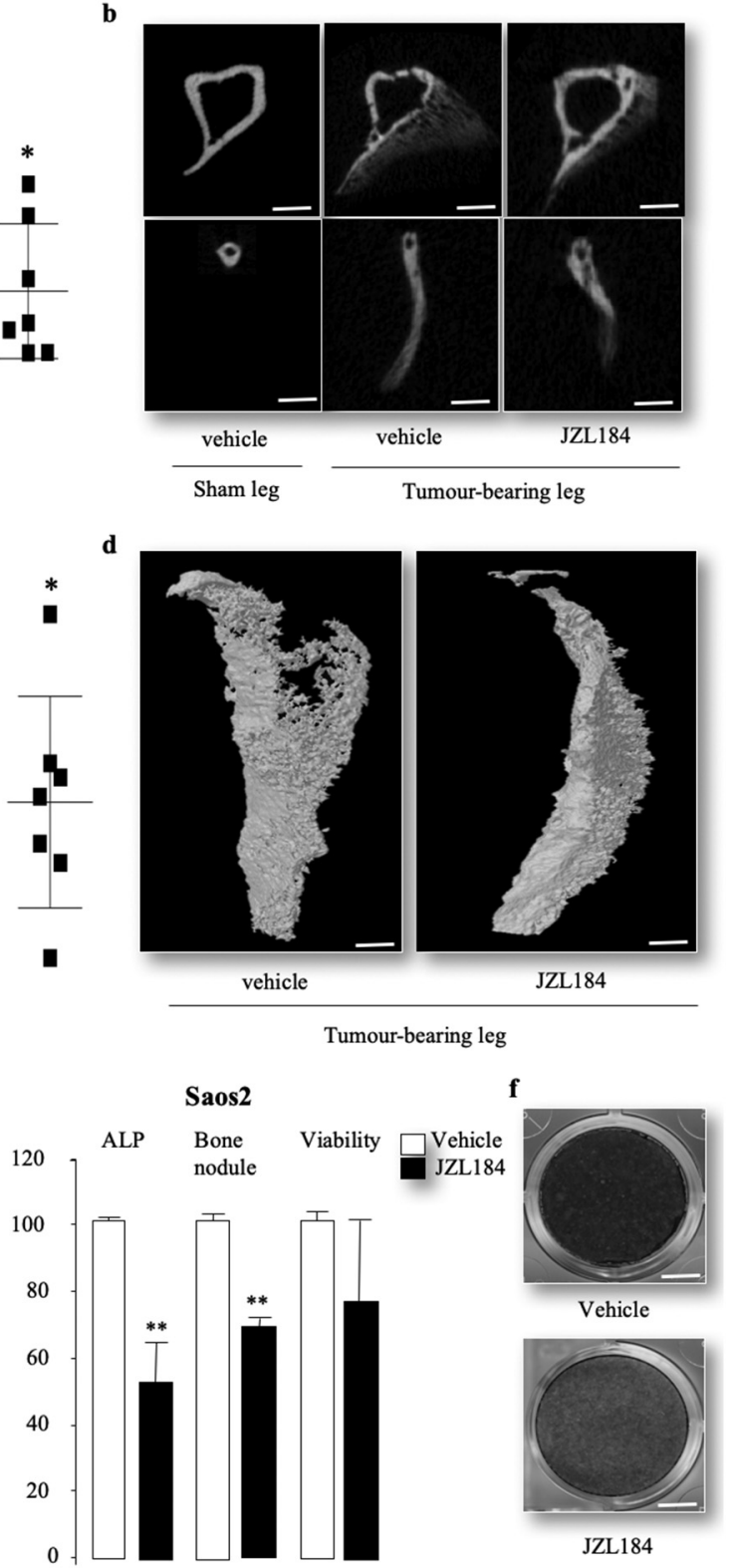

h

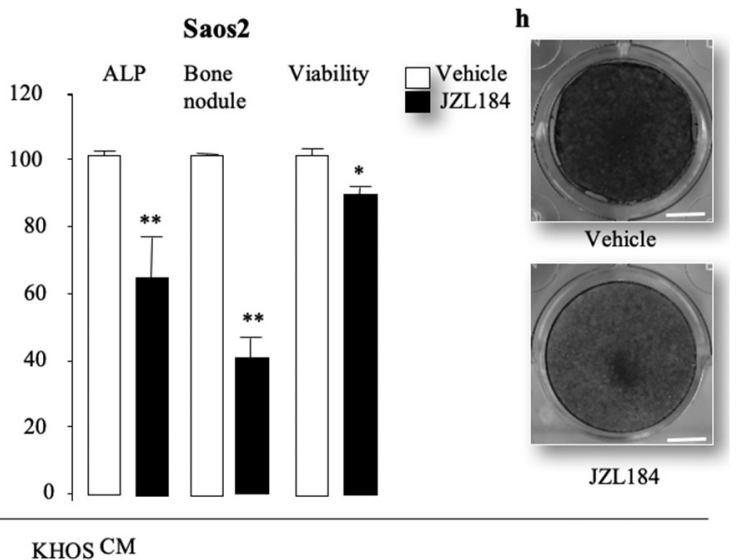

(Fig. 6D, left), osteoclast precursor spreading (Fig. 6D, middle) and maturation into osteoclasts (Fig. 6D, right). In the absence of RANKL, 2AG increased macrophage number cultured in M-CSF (100 ng/ml) alone in a concentration dependent manner (Fig. 6E).

\section{Discussion}

Previous studies have implicated MAGL in cancer and its metabolites $2 \mathrm{AG}$ and free fatty acids in the regulation of bone metabolism $[2,5,6]$. 
However, the role of MAGL in the regulation of skeletal complications related to primary and secondary cancer in bone remains unknown. Here, we provide evidence to show that the verified MAGL inhibitor JZL184 reduces the initiation and progression of bone metastasis, skeletal tumour growth and osteolysis in mouse models of osteosarcoma and prostate or breast cancers. Using in vitro studies in cancer and bone cell cultures, we first show that both stable knockdown and pharmacological inhibition of MAGL reduced the ability of osteotropic breast and prostate cancer cells to migrate, invade and stimulate osteoclastogenesis - thus suggesting anti-metastatic and anti-osteolytic effects. This led us to hypothesise that inhibition of MAGL in cancer and/or bone cells reduces the metastatic spread and osteolytic features of primary and secondary cancer in bone in the models described.

Nomura and colleagues have shown that MAGL knockdown and pharmacological inhibition using JZL184 reduced tumour growth and metastasis of the PC3 prostate cancer cells in mice $[1,4]$. Here we show that MAGL is expressed by osteotropic sub-clones of the prostate cancer cells PC3 in culture and in the long bone of mice, and systemic administration of JZL184 suppressed the ability of these cells to metastasise to the skeleton, grow and cause osteolysis in mice. Our present data expand on previous observations $[1,4]$ and demonstrate that JZL184 exerts similar anti-metastatic, anti-tumour and bone preservation effects in mouse models of the osteoblastic KHOS and osteolytic MDA-231-BT cells, two established models of primary bone cancer (osteosarcoma) and secondary breast cancer metastasis in bone, respectively. In these models, mice received JZL184 also exhibited reduced cachexia and increased survival. Interestingly, JZL184 treatment significantly enhanced trabecular thickness caused by the injection of the human osteosarcoma KHOS cells. This action of JZL184 was likely due to combined inhibition of osteosarcoma-induced bone loss and reduction of tumour growth by this agent rather than direct stimulation of osteoblast differentiation and activity. In stark contract, JZL184 inhibited the formation of cancer associated bone formation within the tumour body in mice inoculated with KHOS cells, a key feature of osteosarcoma [53]. Consistently, we show that JZL184 inhibited the ability of primary osteoblast and early differentiated osteosarcoma cells Saos 2 to differentiate and to form bone nodule in the presence and absence of the highly metastatic osteosarcoma cells KHOS. We excluded the possibility that toxic effects of JZL184 affects the viability of primary osteoblast, osteoblast-like and osteosarcoma cells since we have not observed any changes in cell number in vitro or in vivo.

A surprising result of this study is administration of JZL184 reduced bone loss in the osteolytic MDA-231 and osteoblastic KHOS models without affecting the number or activity of osteoclasts or osteoblasts in mice. This led us to speculate that bone preservation in these mice was mediated by the combined effects of JZL184 on cancer and host cells of the tumour microenvironment. The evidence for this comes from the data that show that inoculation of MAGL deficient cells produced a modest change or no-significant effect on bone volume in the MDA-231 and KHOS models used (supplementary Fig. S6). These effects are inconsistent with previous study that both MAGL knockdown and pharmacological effects reduced tumour growth in mice $[1,4]$. We can not readily explain this other than to speculate that MAGL inhibition in both cancer and host cells may be needed to exert a significant reduction in many aspects of cancer-induced bone disease in the highly aggressive mouse models described. It is also important to note here that the sub-optimal efficiency in the shRNA-mediated knockdown of MAGL expression in the osteolytic MDA-231-BT (MAGL ${ }^{\mathrm{KD} 1}, 72 \%$ reduction) and osteoblastic KHOS (MAGL ${ }^{\mathrm{KD} 2}, 68 \%$ reduction) cells that were used in our studies may have contributed to the modest effects observed on mice injected with MAGL deficient cells.

MAGL is responsible for the degradation of the most abundant endocannabinoid in bone 2AG [19-25], and previous findings have reported increased the local level of 2AG in the skeleton mimicked the anti-hyperalgesic effect of $2 \mathrm{AG}$ via a $\mathrm{Cnr} 2$ receptor-dependent mechanism [72]. Equipped with the knowledge that MAGL metabolites such as 2AG, AEA and others acts at both classical Cnr1 and 2 receptors bone loss [20-25,28] - albeit with different degree of selectivity $[73,74]$, and inactivation of Cnr1, Cnr2 or both receptors has been found to influence peak bone mass and age-related bone loss [19-25,28-33], we hypothesised that MAGL inhibition induces bone loss via an effect that dependent at least in part on the skeletal cannabinoid receptors. In support of this hypothesis, we have shown that bone marrow cells, osteoblasts and osteoclasts express MAGL and exposure to JZL184 at nanomolar concentrations induces the expression of osteoclast specific genes, enhances RANKL- and osteoblast-induced osteoclast formation and causes bone loss in the presence and absence of osteolytic cancer cells. Most importantly, the latter effect was significantly blunted in mice or calvarial bone organ cultures deficient in both $\mathrm{Cnr} 1$ and $\mathrm{Cnr} 2$ cannabinoid receptors. Interestingly, we have also observed that pre-exposure of calvarial bone and osteolytic cancer cells to the Cnr2 selective inverse agonist AM630 was more effective in blunting the pro-osteolytic action of JZL184 when compared to treatment with the Cnr1 selective AM251. It is tempting to speculate about the involvement of an individual cannabinoid receptor in the proosteolytic action of JZL187 in our experiments since these agents have been previously shown to act on multiple targets at concentrations tested. The fact that JZL184 failed to completely reverse osteolysis in calvarial bone from Cnr1/2 knockout mice when compared to wild type control have led us to examine the level of tumour-derived proinflammatory mediators that we and others have reported to regulate the interaction between osteotropic cancer cells and osteoclasts $[8-10,15,46,75]$. These experiments revealed that JZL184 regulates the production of tumour-derived osteolytic and pro-osteoblastic factors. The marked increase in the levels of osteolytic factors such as VEGF and resistin and may account for JZL184 pro-osteoclast action and/or its failure to inhibit osteoclastogenesis in the presence of cancer cells in mice. VEGF, resistin and other detected factors including osteopontin are known to enhance the proliferation, maturation and RANKL by osteoblasts [59,76], a possible mechanism for MDA-231-BT and JZL184 associated bone loss in the model described. Another plausible mechanism may involve the reduction of host- and/or tumour-derived free fatty acids, key metabolites of MAGL that have been shown to regulate bone growth and remodelling [77-79]. Receptors and fatty acid sensors are expressed by osteoclasts, osteoblasts and their precursors [80,81], and a number of in vitro studies have shown that FFA reduce or stimulate osteoclastogenesis depending on cell type and culture conditions $[80,81]$. Another intriguing finding is both JZL184 and 2AG stimulated the growth of M-CSF dependent bone marrow macrophage at micromolar concentrations that inhibited osteoclast formation. Bearing in mind that a number of studies have previously reported that 2AG

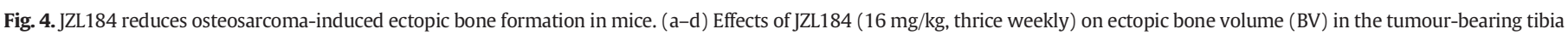

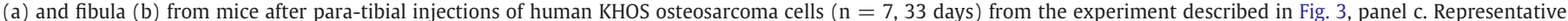

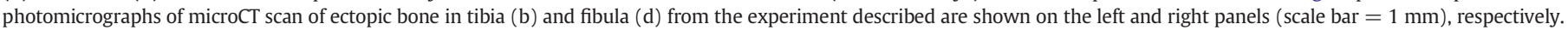

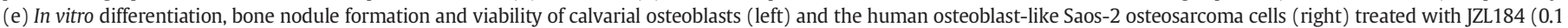

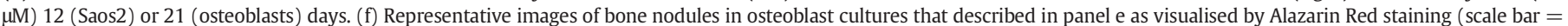

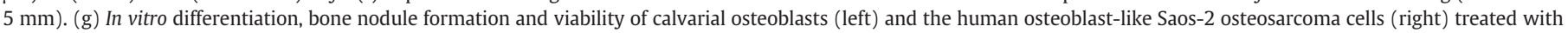

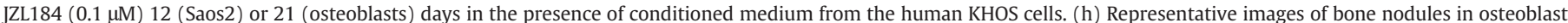

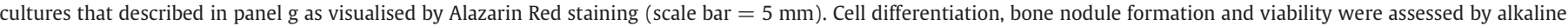
phosphatase, Alazarin Red and AlamarBlue assays, respectively $(n=3)$. Data are mean $\pm \mathrm{SD},{ }^{*} p<.05,{ }^{* *}, p<.01$. 


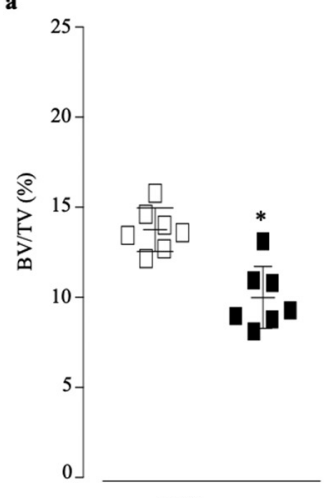

Wild type

d

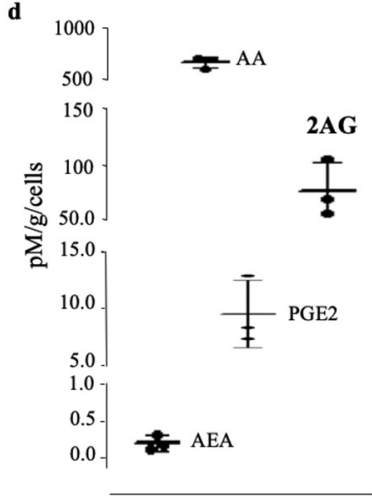

MDA-231-BT CM b

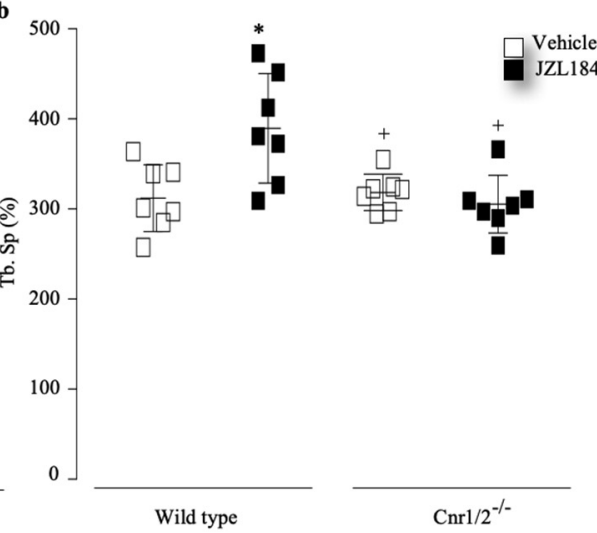

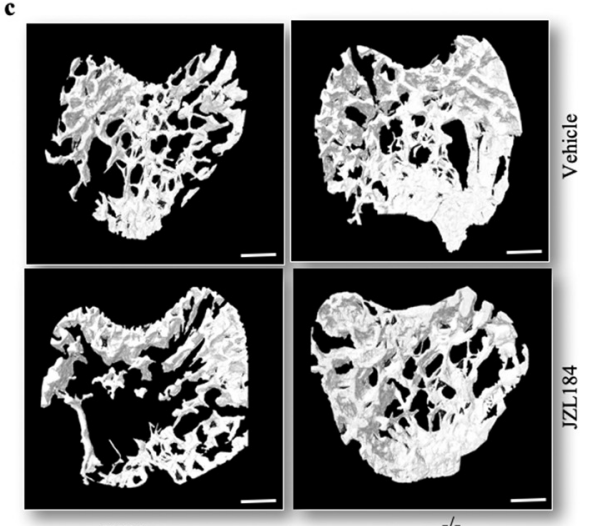

Wild type

$\mathrm{Cnr} 1 / 2^{-/-}$

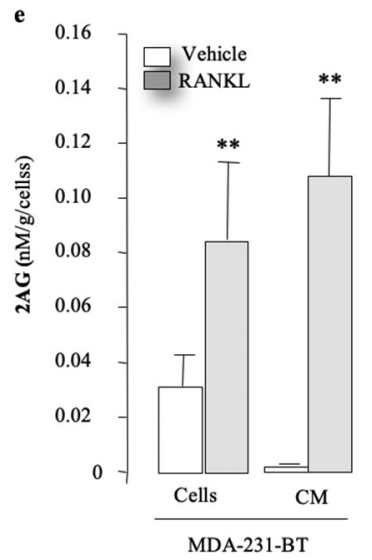

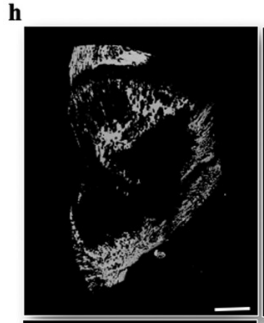

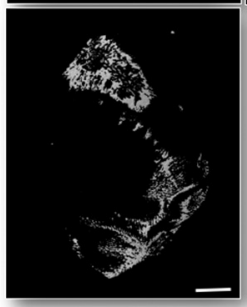

Wild type

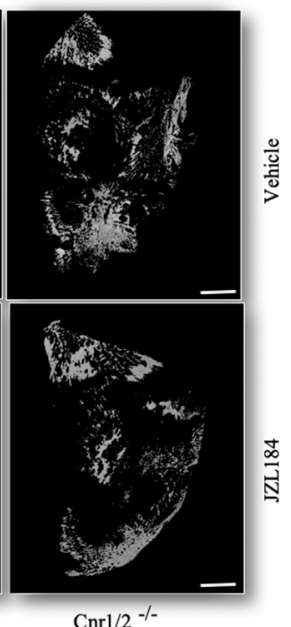

i
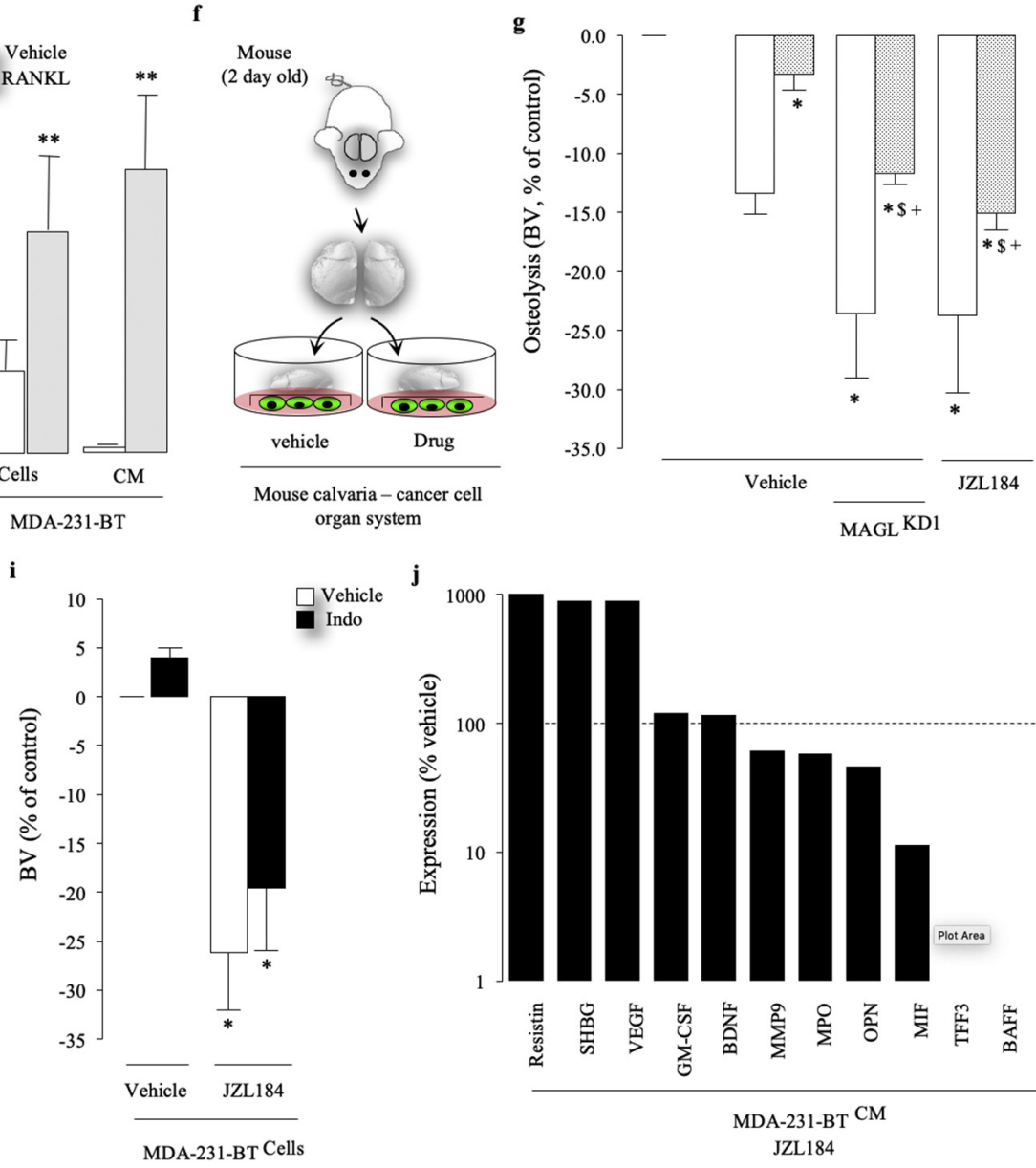

Wild type

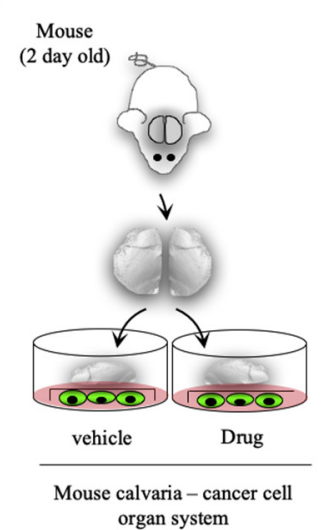

$\mathrm{Cnrl} / 2^{-/-}$

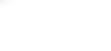

j 1000

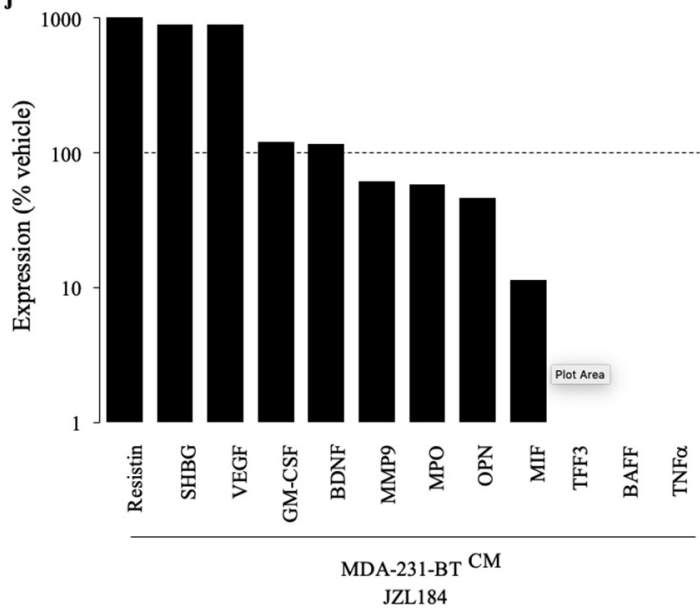

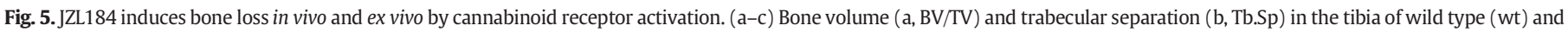

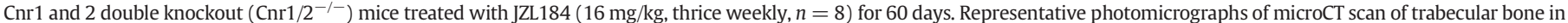

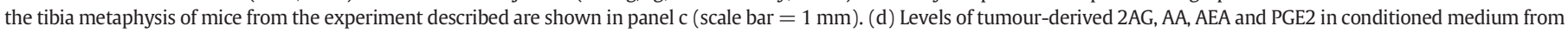

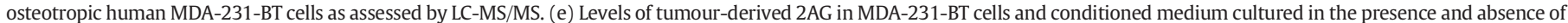

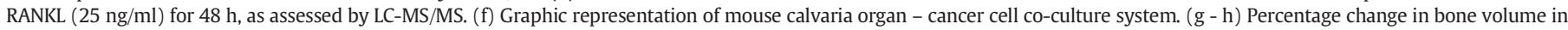

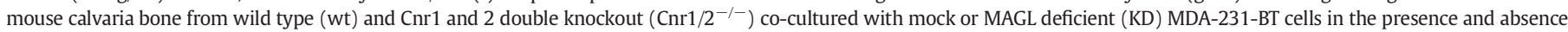

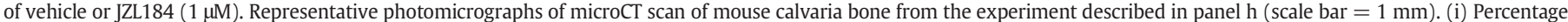

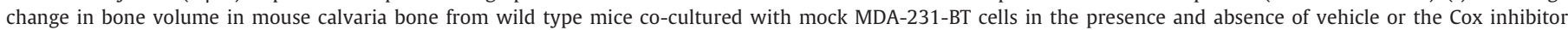

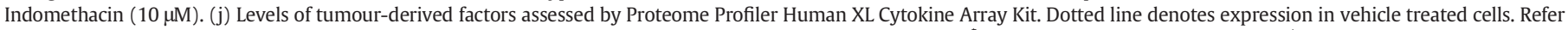
to text for abbreviations. Data are mean $\pm \mathrm{SD},{ }^{*} \mathrm{p}<.05$ and ${ }^{* *} \mathrm{p}<.01$ from control; $+\mathrm{p}<.05$ from wild type (wt); ${ }^{\$} p<.00$ from vehicle treated Cnr $1 / 2^{-/-}$.

enhances macrophage function [82-85], it is tempting to speculate that macrophage-derived $2 \mathrm{AG}$ - alone or in cooperation with a cocktail of pro-inflammatory mediators - inhibit osteoclast differentiation to maintain macrophage function in our models [86]. A plethora of other members of the serine hydrolase family of enzymes, intracellular signalling pathways including NFKB and $\beta$-catenin and physiological mediators such as nitric oxide and eicosanoids have been implicated in the action of JZL184 [52,87-89]. Since all of these mediators have an inflammatory and immune component, it is possible they may play a role in the effects of JZL184 on initiation and progression of cancer associated bone disease in the models described. Thus, further mechanistic studies are warranted and on-going. 

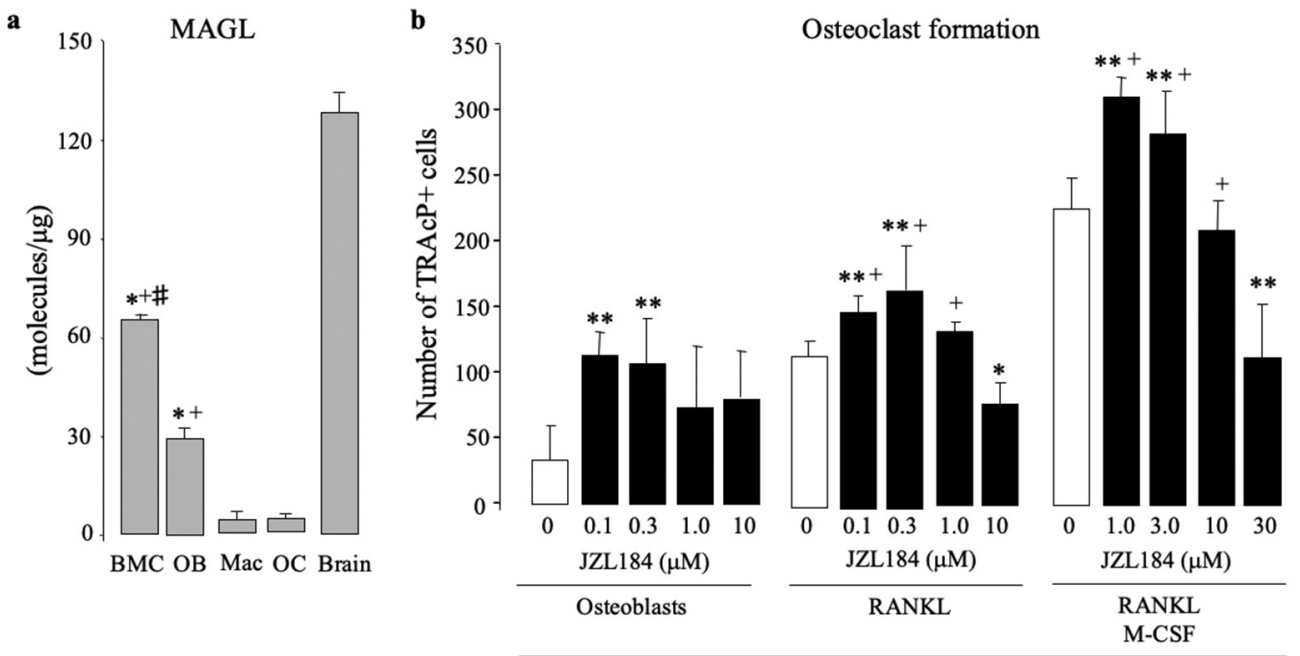

Bone marrow cells (BMC)

c Pre-osteoclast spreading
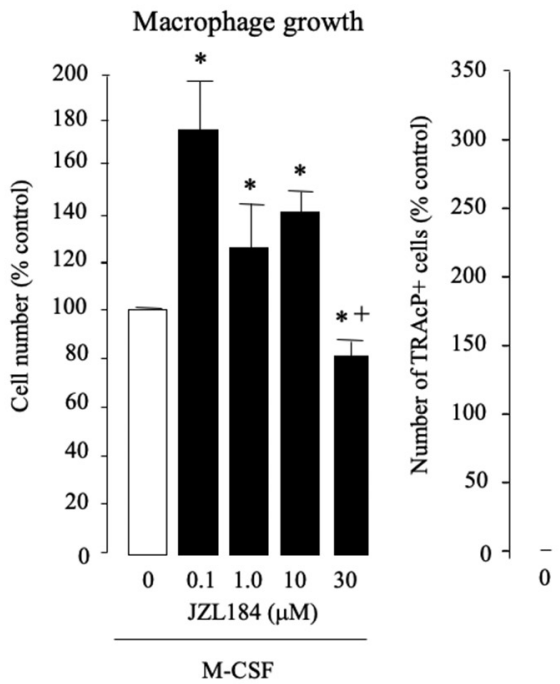

Osteoclast formation

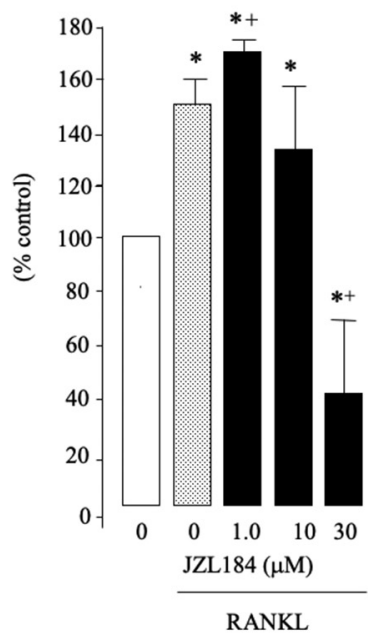

d

Osteoclast specific genes

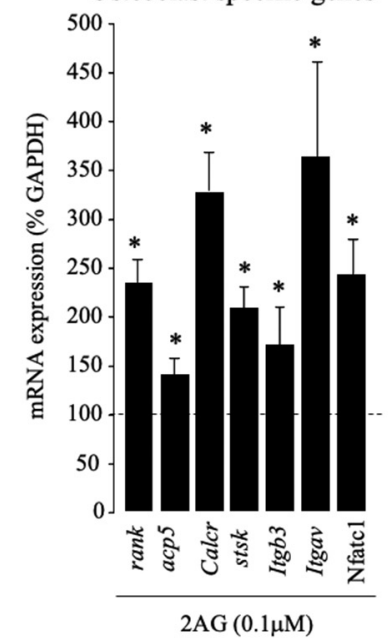

e Pre-osteoclast spreading

Osteoclast formation
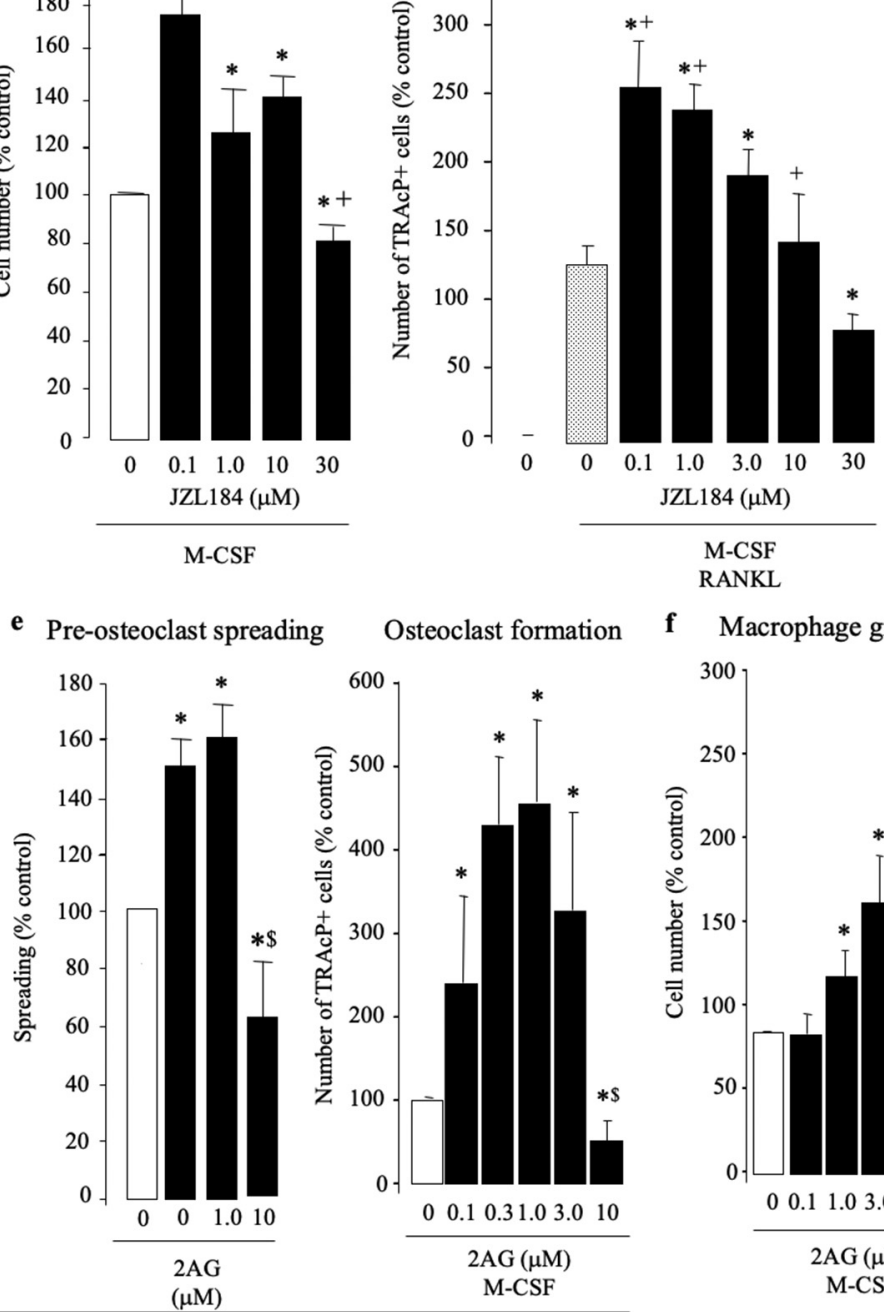

f Macrophage growth

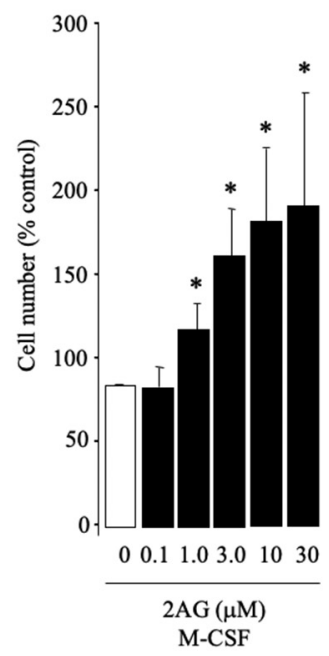

RANKL

Collectively, the results presented in this study extend our and others previous findings on the role endocannabinoids and their receptors in the skeleton [17-19], and show that the verified MAGL inhibitor JZL184 exerts anti-tumour and anti-metastatic effects, alleviates cachexia and prolongs survival in the cancer models described, but it causes bone loss in healthy mice. When combined with previous study [1,4], these findings reveal a complex function of MAGL in the regulation of normal and cancer-related bone remodelling (Fig. 7), offer a 


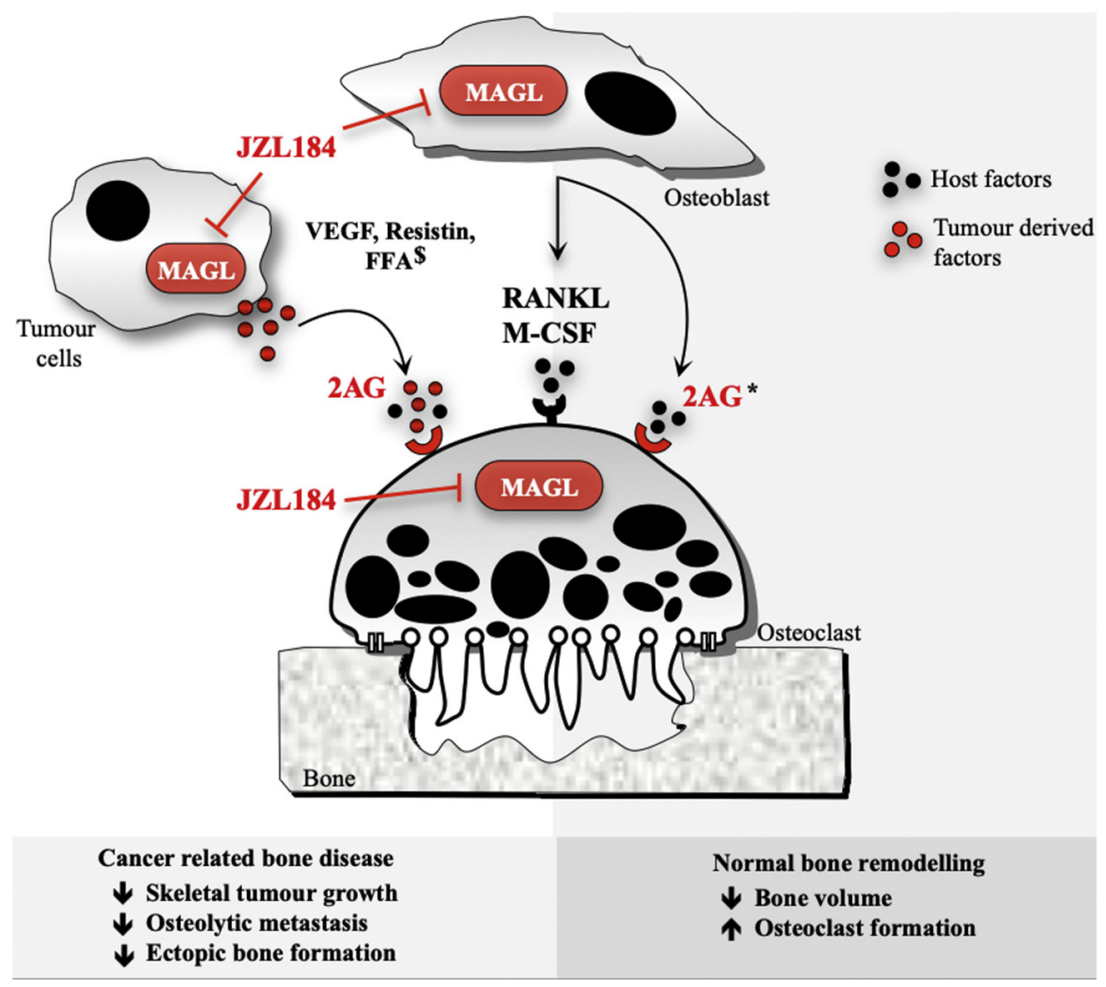

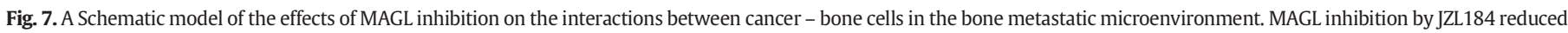

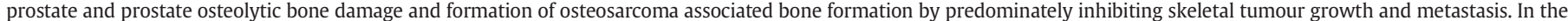

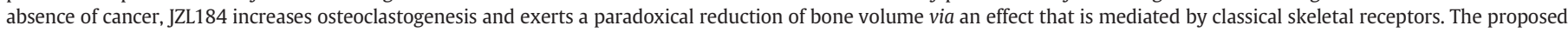

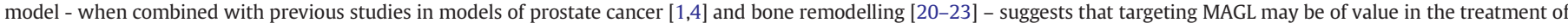

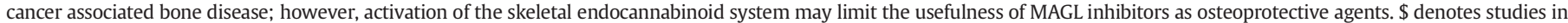
models of prostate cancer $[1,4]$ and * denotes studies in models of bone remodelling [20-23].

new insight into the role of the skeletal endocannabinoid system in the crosstalk between cancer and bone cells and implies that MAGL inhibitors such as JZL184, or its novel analogues, may be of value in the treatment of skeletal complications associated with primary bone cancer and bone metastasis, but caution against the potential detrimental effects of therapeutic targeting of MAGL for the treatment of bone disorders. For that, further mechanistic studies are needed.

\section{Declaration of interests}

A.I. Idris and S.H. Ralston are inventors on a patent concerning the use of cannabinoid receptor ligands for the treatments of bone diseases. Other authors declare no conflict of interest.

\section{Funding sources}

The studies described were partly supported by funding from Cancer Research UK Development Fund (Edinburgh Cancer Research Centre, University of Edinburgh) and a programme grant from Arthritis Research UK.

\section{Author contributions}

Silvia Marino, Daniëlle de Ridder, Ryan T. Bishop, Nathalie Renema, Marco Ponzetti, Antonia Sophocleous, Mattia Capulli, Abdullah Aljeffery, Giovana Carrasco, Marianela Dalghi Gens, Asim Kheeger, are involved in experimental and analysis; Stuart H. Ralston, Jürg Gertsch, Francois Lamoureux, Dominique Heymann, Nadia Rucci are involved in conception and analysis, and Aymen I. Idris is involved in conception, experimental, analysis and writing.

\section{Acknowledgments}

I, AI Idris, would like to thank the postgraduate students, postdoctoral researchers and laboratory leaders who dedicated their spare time and resources to generate the data for the present manuscript, and acknowledge the invaluable suggestions and constructive comments by the anonymous scientists who reviewed our unsuccessful cannabinoid-related grant applications over the past 9 years on behalf of a number of funding bodies.

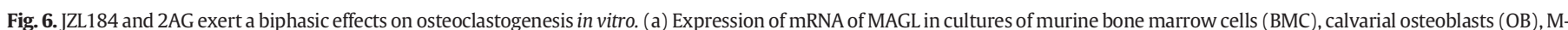

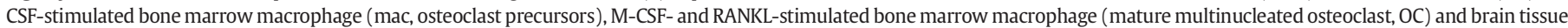

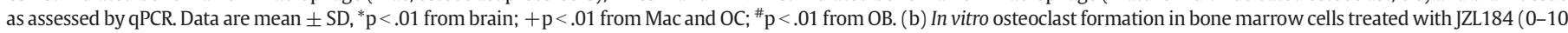

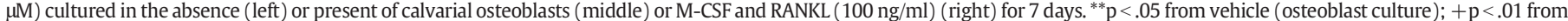

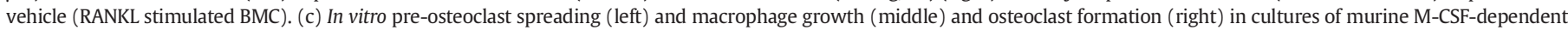

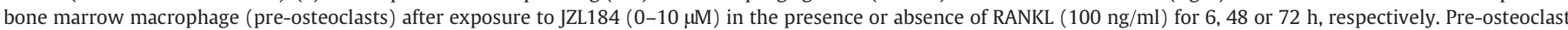

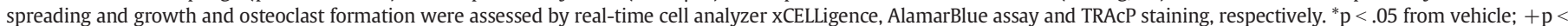

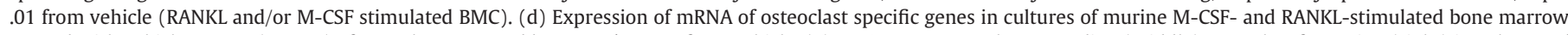

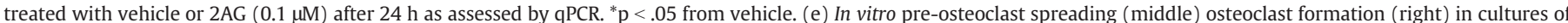

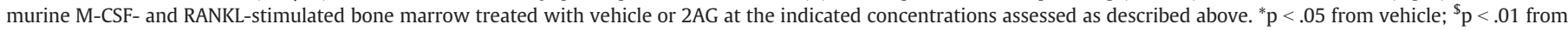

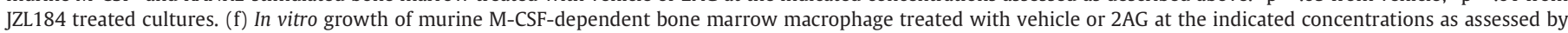
AlamarBlue assay. ${ }^{*} \mathrm{p}<.05$ from vehicle. 


\section{Appendix A. Supplementary data}

Supplementary data to this article can be found online at https://doi. org/10.1016/j.ebiom.2019.05.048.

\section{References}

[1] Mulvihill MM, Nomura DK. Therapeutic potential of monoacylglycerol lipase inhibitors. Life Sci 2013;92(8-9):492-7.

[2] Pisanti S, Picardi P, D'Alessandro A, Laezza C, Bifulco M. The endocannabinoid signaling system in cancer. Trends Pharmacol Sci 2013;34(5):273-82.

[3] Fowler CJ. Monoacylglycerol lipase - a target for drug development? Br J Pharmacol 2012;166(5):1568-85.

[4] Nomura DK, Lombardi DP, Chang JW, et al. Monoacylglycerol lipase exerts dual control over endocannabinoid and fatty acid pathways to support prostate cancer. Chem Biol 2011;18(7):846-56.

[5] Fraguas-Sanchez AI, Martin-Sabroso C, Torres-Suarez AI. Insights into the effects of the endocannabinoid system in cancer: a review. Br J Pharmacol 2018;175(13): 2566-80.

[6] Nithipatikom K, Endsley MP, Isbell MA, Wheelock CE, Hammock BD, Campbell WB. A new class of inhibitors of 2-arachidonoylglycerol hydrolysis and invasion of prostate cancer cells. Biochem Biophys Res Commun 2005;332(4):1028-33.

[7] Gjerstorff MF, Benoit VM, Laenkholm AV, Nielsen O, Johansen LE, Ditzel HJ. Identification of genes with altered expression in medullary breast cancer vs. ductal breast cancer and normal breast epithelia. Int J Oncol 2006;28(6):1327-35.

[8] Mundy GR. Metastasis to bone: causes, consequences and therapeutic opportunities. NatRevCancer 2002;2(8):584-93.

[9] Roodman GD. Mechanisms of bone metastasis. NEnglJMed 2004;350(16):1655-64.

[10] Coleman RE. Metastatic bone disease: clinical features, pathophysiology and treatment strategies. Cancer Treat Rev 2001;27(3):165-76.

[11] Yoneda T, Hiraga T. Crosstalk between cancer cells and bone microenvironment in bone metastasis. BiochemBiophysResCommun 2005;328(3):679-87.

[12] Broadhead ML, Clark JC, Myers DE, Dass CR, Choong PF. The molecular pathogenesis of osteosarcoma: a review. Sarcoma 2011;2011:959248.

[13] Bienz M, Saad F. Management of bone metastases in prostate cancer: a review. Curr Opin Support Palliat Care 2015;9(3):261-7.

[14] UK CR. http://www.cancerresearchuk.org/health-professional/cancer-statistics/ statistics-by-cancer-type/prostate-cancer-heading-One.

[15] Siclari VA, Guise TA, Chirgwin JM. Molecular interactions between breast cancer cells and the bone microenvironment drive skeletal metastases. Cancer Metastasis Rev 2006;25(4):621-33.

[16] Brown HK, Schiavone K, Gouin F, Heymann MF, Heymann D. Biology of bone sarcomas and new therapeutic developments. Calcif Tissue Int 2018;102(2):174-95.

[17] Bab I, Zimmer A, Melamed E. Cannabinoids and the skeleton: from marijuana to reversal of bone loss. AnnMed 2009:1-8.

[18] Marino S, Idris AI. Emerging therapeutic targets in cancer induced bone disease: a focus on the peripheral type 2 cannabinoid receptor. Pharmacol Res 2017;119: 391-403.

[19] Idris AI, Ralston SH. Cannabinoids and bone: friend or foe? Calcif Tissue Int 2010;87 (4):285-97.

[20] Bab I, Ofek O, Tam J, Rehnelt J, Zimmer A. Endocannabinoids and the regulation of bone metabolism. JNeuroendocrinol 2008;20(Suppl. 1):69-74.

[21] Tam J, Trembovler V, Di MV, et al. The cannabinoid CB1 receptor regulates bone formation by modulating adrenergic signaling. FASEB J 2008;22(1):285-94.

[22] Tam J, Ofek O, Fride E, et al. Involvement of neuronal cannabinoid receptor, CB1, in regulation of bone mass and bone Remodeling. MolPharmacol 2006;70(3):786-92

[23] Whyte LS, Ford L, Ridge SA, Cameron GA, Rogers MJ, Ross RA. Cannabinoids and bone: endocannabinoids modulate human osteoclast function in vitro. $\mathrm{Br} \mathrm{J}$ Pharmacol 2012;165(82):2584-97.

[24] Rossi F, Siniscalco D, Luongo L, et al. The endovanilloid/endocannabinoid system in human osteoclasts: possible involvement in bone formation and resorption. Bone 2009;44(3):476-84.

[25] Smith M, Wilson R, O'Brien S, Tufarelli C, Anderson SI, O'Sullivan SE. The effects of the endocannabinoids anandamide and 2-arachidonoylglycerol on human osteoblast proliferation and differentiation. PLoS One 2015;10(9):e0136546.

[26] Pertwee RG, Ross RA. Cannabinoid receptors and their ligands. Prostaglandins Leukot Essent Fatty Acids 2002;66(2-3):101-21.

[27] Di Marzo V. Targeting the endocannabinoid system: to enhance or reduce? Nat Rev Drug Discov 2008;7(5):438-55.

[28] Ofek O, Karsak M, Leclerc N, et al. Peripheral cannabinoid receptor, CB2, regulates bone mass. ProcNatlAcadSciUSA 2006;103(3):696-701.

[29] Sophocleous A, Landao-Bassonga E, Van't Hof RJ, Idris AI, Ralston SH. The type 2 cannabinoid receptor regulates bone mass and ovariectomy-induced bone loss by affecting osteoblast differentiation and bone formation. Endocrinology 2011;152(6): 2141-9.

[30] Idris AI, Sophocleous A, Landao-Bassonga E, et al. Cannabinoid receptor type 1 protects against age-related osteoporosis by regulating osteoblast and adipocyte differentiation in marrow stromal cells. Cell Metab 2009;10(2):139-47.

[31] Idris AI, Sophocleous A, Landao-Bassonga E, van't Hof RJ, Ralston SH. Regulation of bone mass, osteoclast function, and ovariectomy-induced bone loss by the type 2 cannabinoid receptor. Endocrinology 2008;149(11):5619-26.

[32] Idris AI, Van 't Hof RJ, Greig IR, et al. Regulation of bone mass, bone loss and osteoclast activity by cannabinoid receptors. NatMed 2005;11(7):774-9.
[33] Sophocleous A, Marino S, Kabir D, Ralston SH, Idris AI. Combined deficiency of the Cnr1 and Cnr2 receptors protects against age-related bone loss by osteoclast inhibition. Aging Cell 2017;16(5):1051-61.

[34] Rucci N, E R, Ficorella C, et al. In vivo bone metastases, osteoclastogenic ability, and phenotypic characterization of human breast cancer cells. Bone 2004;34(4): 697-709.

[35] Logan JG, Sophocleous A, Marino S, Muir M, Brunton VG, Idris AI. Selective tyrosine kinase inhibition of insulin-like growth factor-1 receptor inhibits human and mouse breast cancer-induced bone cell activity, bone remodeling, and osteolysis. J Bone Min Res 2013;28(5):1229-42.

[36] Rose AA, Pepin F, Russo C, Abou Khalil JE, Hallett M, Siegel PM. Osteoactivin promotes breast cancer metastasis to bone. Mol Cancer Res 2007;5(10):1001-14.

[37] Wang N, Docherty F, Brown HK, et al. Mitotic quiescence, but not unique "stemness," marks the phenotype of bone metastasis-initiating cells in prostate cancer. FASEB J 2015;29(8):3141-50.

[38] Erben RG, Glosmann M. Histomorphometry in rodents. Methods Mol Biol 2019; 1914:411-35.

[39] Dempster DW, Compston JE, Drezner MK, et al. Standardized nomenclature, symbols, and units for bone histomorphometry: a 2012 update of the report of the ASBMR Histomorphometry nomenclature committee. J Bone Min Res 2013;28(1): 2-17.

[40] Orriss IR, Taylor SE, Arnett TR. Rat osteoblast cultures. Methods Mol Biol 2012;816: 31-41.

[41] Gloeckner H, Jonuleit T, Lemke HD. Monitoring of cell viability and cell growth in a hollow-fiber bioreactor by use of the dye Alamar Blue. J Immunol Methods 2001; 252(1-2):131-8.

[42] Marino S, Logan JG, Mellis D, Capulli M. Generation and culture of osteoclasts. Bone Key Rep 2014;3:570.

[43] Van't Hof RJ. Osteoclast formation in the mouse coculture assay. In: Helfrich MH, Ralston SH, editors. Bone research protocols. Totowa: Humana Press; 2003. p. $145-52$

[44] Atienza JM, Zhu J, Wang X, Xu X, Abassi Y. Dynamic monitoring of cell adhesion and spreading on microelectronic sensor arrays. JBiomolScreen 2005;10(8): 795-805.

[45] Campbell GM, Sophocleous A. Quantitative analysis of bone and soft tissue by microcomputed tomography: applications to ex vivo and in vivo studies. Bone Key Rep 2014;3:564.

[46] Marino S, Bishop RT, Logan JG, Mollat P, Idris AI. Pharmacological evidence for the bone-autonomous contribution of the NFkappaB/beta-catenin axis to breast cancer related osteolysis. Cancer Lett 2017;410:180-90.

[47] Schlosburg JE, Blankman JL, Long JZ, et al. Chronic monoacylglycerol lipase blockade causes functional antagonism of the endocannabinoid system. Nat Neurosci 2010;13 (9):1113-9.

[48] Long JZ, Li W, Booker L, et al. Selective blockade of 2-arachidonoylglycerol hydrolysis produces cannabinoid behavioral effects. Nat Chem Biol 2009;5(1):37-44.

[49] Chen R, Zhang J, Wu Y, et al. Monoacylglycerol lipase is a therapeutic target for Alzheimer's disease. Cell Rep 2012;2(5):1329-39.

[50] Long JZ, Nomura DK, Cravatt BF. Characterization of monoacylglycerol lipase inhibition reveals differences in central and peripheral endocannabinoid metabolism. Chem Biol 2009;16(7):744-53.

[51] Nomura DK, Morrison BE, Blankman JL, et al. Endocannabinoid hydrolysis generates brain prostaglandins that promote neuroinflammation. Science 2011;334(6057): 809-13.

[52] Qin H, Ruan ZH. The role of monoacylglycerol lipase (MAGL) in the cancer progress. Cell Biochem Biophys 2014;70(1):33-6.

[53] Kim SJ, Choi JA, Lee SH, et al. Imaging findings of extrapulmonary metastases of osteosarcoma. Clin Imaging 2004;28(4):291-300.

[54] Geller DS, Gorlick R. Osteosarcoma: a review of diagnosis, management, and treatment strategies. Clin Adv Hematol Oncol 2010;8(10):705-18.

[55] Lamoureux F, Baud'huin M, Rodriguez Calleja L, et al. Selective inhibition of BET bromodomain epigenetic signalling interferes with the bone-associated tumour vicious cycle. Nat Commun 2014;5:3511.

[56] Sophocleous A, Idris AI, Ralston SH. Genetic background modifies the effects of type 2 cannabinoid receptor deficiency on bone mass and bone turnover. Calcif Tissue Int 2014;94(3):259-68.

[57] Sophocleous A, Sims AH, Idris AI, Ralston SH. Modulation of strain-specific differences in gene expression by cannabinoid type 2 receptor deficiency. Calcif Tissue Int 2014;94(4):423-32.

[58] Sophocleous A, Robertson R, Ferreira NB, McKenzie J, Fraser WD, Ralston SH. Heavy cannabis use is associated with low bone mineral density and an increased risk of fractures. Am J Med 2017;130(2):214-21.

[59] Thommesen L, Stunes AK, Monjo M, et al. Expression and regulation of resistin in osteoblasts and osteoclasts indicate a role in bone metabolism. J Cell Biochem 2006;99 (3):824-34.

[60] Isowa S, Shimo T, Ibaragi S, et al. PTHrP regulates angiogenesis and bone resorption via VEGF expression. Anticancer Res 2010;30(7):2755-67.

[61] Hu K, Olsen BR. Osteoblast-derived VEGF regulates osteoblast differentiation and bone formation during bone repair. J Clin Invest 2016;126(2):509-26.

[62] Sun CY, Chu ZB, She XM, et al. Brain-derived neurotrophic factor is a potential osteoclast stimulating factor in multiple myeloma. Int J Cancer J Int Cancer 2012;130(4): 827-36.

[63] Lee MS, Kim HS, Yeon JT, et al. GM-CSF regulates fusion of mononuclear osteoclasts into bone-resorbing osteoclasts by activating the Ras/ERK pathway. J Immunol 2009;183(5):3390-9.

[64] Hemingway F, Taylor R, Knowles HJ, Athanasou NA. RANKL-independent human osteoclast formation with APRIL, BAFF, NGF, IGF I and IGF II. Bone 2011;48(4):938-44. 
[65] Movila A, Ishii T, Albassam A, et al. Macrophage migration inhibitory factor (MIF) supports homing of osteoclast precursors to peripheral Osteolytic lesions. J Bone Min Res 2016;31(9):1688-700.

[66] Nbžpktbr Radić. Trefoil factor family protein 3 affects cancellous bone formation in the secondary centers of ossification of mouse tibiae. Periodicum Biologorum 2015;117(1):59-64.

[67] Franzen A, Hultenby K, Reinholt FP, Onnerfjord P, Heinegard D. Altered osteoclast development and function in osteopontin deficient mice. J Orthop Res 2008;26(5): 721-8.

[68] Panagopoulos V, Liapis V, Zinonos I, et al. Peroxidase enzymes inhibit osteoclast differentiation and bone resorption. Mol Cell Endocrinol 2017;440:8-15

[69] Thomson BM, Mundy GR, Chambers TJ. Tumor necrosis factors alpha and beta induce osteoblastic cells to stimulate osteoclastic bone resorption. J Immunol 1987; 138(3):775-9.

[70] Atanga E, Dolder S, Dauwalder T, Wetterwald A, Hofstetter W. TNFalpha inhibits the development of osteoclasts through osteoblast-derived GM-CSF. Bone 2011;49(5): 1090-100.

[71] Gilbert L, He X, Farmer P, et al. Inhibition of osteoblast differentiation by tumor necrosis factor-alpha. Endocrinology 2000;141(11):3956-64.

[72] Khasabova IA, Chandiramani A, Harding-Rose C, Simone DA, Seybold VS. Increasing 2 -arachidonoyl glycerol signaling in the periphery attenuates mechanical hyperalgesia in a model of bone cancer pain. Pharmacol Res 2011;64(1):60-7.

[73] Matias I, Pochard P, Orlando P, Salzet M, Pestel J, Di MV. Presence and regulation of the endocannabinoid system in human dendritic cells. EurJBiochem 2002;269(15): 3771-8.

[74] Mechoulam R. Plant cannabinoids: a neglected pharmacological treasure trove. $\mathrm{Br} \mathrm{J}$ Pharmacol 2005;146(7):913-5.

[75] Kang Y, Siegel PM, Shu W, et al. A multigenic program mediating breast cancer metastasis to bone. Cancer Cell 2003;3(6):537-49.

[76] Nakai T, Yoshimura Y, Deyama Y, Suzuki K, lida J. Mechanical stress up-regulates RANKL expression via the VEGF autocrine pathway in osteoblastic MC3T3-E1 cells. Mol Med Rep 2009;2(2):229-34.

[77] Verma S, Rajaratnam JH, Denton J, Hoyland JA, Byers RJ. Adipocytic proportion of bone marrow is inversely related to bone formation in osteoporosis. J Clin Pathol 2002;55(9):693-8.
[78] Cui LH, Shin MH, Chung EK, et al. Association between bone mineral densities and serum lipid profiles of pre- and post-menopausal rural women in South Korea. Osteoporos Int 2005;16(12):1975-81.

[79] Parhami F, Tintut Y, Beamer WG, Gharavi N, Goodman W, Demer LL. Atherogenic high-fat diet reduces bone mineralization in mice. J Bone Min Res 2001;16(1): $182-8$.

[80] Oh SR, Sul OJ, Kim YY, et al. Saturated fatty acids enhance osteoclast survival. J Lipid Res 2010;51(5):892-9.

[81] Cornish J, MacGibbon A, Lin JM, et al. Modulation of osteoclastogenesis by fatty acids. Endocrinology 2008;149(11):5688-95.

[82] Lee JH, Hou X, Kummari E, Borazjani A, Edelmann MJ, Ross MK. Endocannabinoid hydrolases in avian HD11 macrophages identified by chemoproteomics: inactivation by small-molecule inhibitors and pathogen-induced downregulation of their activity. Mol Cell Biochem 2018;444(1-2):125-41.

[83] Chuchawankul S, Shima M, Buckley NE, Hartmann CB, McCoy KL. Role of cannabinoid receptors in inhibiting macrophage costimulatory activity. Int Immunopharmacol 2004;4(2):265-78.

[84] Taylor L, Christou I, Kapellos TS, et al. Primary macrophage Chemotaxis induced by cannabinoid receptor 2 agonists occurs independently of the CB2 receptor. Sci Rep 2015;5:10682.

[85] Mai P, Tian L, Yang L, Wang L, Yang L, Li L. Cannabinoid receptor 1 but not 2 mediates macrophage phagocytosis by G(alpha)i/o /RhoA/ROCK signaling pathway. J Cell Physiol 2015;230(7):1640-50.

[86] Lampiasi N, Russo R, Zito F. The alternative faces of macrophage generate osteoclasts. Biomed Res Int 2016;2016:9089610.

[87] Zhu W, Zhao Y, Zhou J, et al. Monoacylglycerol lipase promotes progression of hepatocellular carcinoma via NF-kappaB-mediated epithelial-mesenchymal transition. J Hematol Oncol 2016;9(1):127.

[88] Kerr DM, Harhen B, Okine BN, Egan LJ, Finn DP, Roche M. The monoacylglycerol lipase inhibitor JZL184 attenuates LPS-induced increases in cytokine expression in the rat frontal cortex and plasma: differential mechanisms of action. Br J Pharmacol 2013;169(4):808-19.

[89] Crowe MS, Leishman E, Banks ML, et al. Combined inhibition of monoacylglycerol lipase and cyclooxygenases synergistically reduces neuropathic pain in mice. $\mathrm{Br} \mathrm{J}$ Pharmacol 2015;172(7):1700-12. 